\title{
Evaluation of Wearable Technology in Dementia: A Systematic Review and Meta-Analysis
}

\author{
Alanna C. Cote ${ }^{1,2 \dagger}$, Riley J. Phelps ${ }^{1 \dagger}$, Nina Shaafi Kabiri ${ }^{1}$, Jaspreet S. Bhangu ${ }^{1,3 *}$ and \\ Kevin "Kip" Thomas" \\ ${ }^{1}$ Department of Anatomy and Neurobiology, Boston University Medical Center, Boston, MA, United States, ${ }^{2}$ Department of \\ Genetics and Genomic Sciences, Icahn School of Medicine at Mount Sinai, New York, NY, United States, ${ }^{3}$ Division of \\ Geriatric Medicine, Department of Medicine, Western University, London, ON, Canada
}

OPEN ACCESS

Edited by:

Jon Irazusta,

University of the Basque

Country, Spain

Reviewed by:

Tomasz Grodzicki,

Jagiellonian University Medical

College, Poland

Javier Santabarbara,

University of Zaragoza, Spain

${ }^{*}$ Correspondence:

Jaspreet S. Bhangu

jbhangu@bu.edu

${ }^{\dagger}$ These authors have contributed equally to this work

Specialty section:

This article was submitted to

Geriatric Medicine,

a section of the journal

Frontiers in Medicine

Received: 27 September 2019 Accepted: 30 November 2020 Published: 11 January 2021

Citation:

Cote AC, Phelps RJ, Kabiri NS, Bhangu JS and Thomas KK (2021) Evaluation of Wearable Technology in Dementia: A Systematic Review and Meta-Analysis. Front. Med. 7:501104 doi: 10.3389/fmed.2020.501104
Background: The objective of this analysis was to systematically review studies employing wearable technology in patients with dementia by quantifying differences in digitally captured physiological endpoints.

Methods: This systematic review and meta-analysis was based on web searches of Cochrane Database, Psyclnfo, Pubmed, Embase, and IEEE between October 25-31st, 2017. Observational studies providing physiological data measured by wearable technology on participants with dementia with a mean age $\geq 50$. Data were extracted according to PRISMA guidelines and methodological quality assessed independently using Downs and Black criteria. Standardized mean differences between cases and controls were estimated using random-effects models.

Results: Forty-eight studies from 18,456 screened abstracts (Dementia: $n=2,516$, Control: $n=1,224)$ met inclusion criteria for the systematic review. Nineteen of these studies were included in one or multiple meta-analyses (Dementia: $n=617$, Control: $n=406$ ). Participants with dementia demonstrated lower levels of daily activity (standardized mean difference (SMD), $-1.60 ; 95 \% \mathrm{Cl},-2.66$ to -0.55$)$, decreased sleep efficiency (SMD, $-0.52 ; 95 \% \mathrm{Cl},-0.89$ to -0.16$)$, and greater intradaily circadian variability (SMD, $0.46 ; 95 \% \mathrm{Cl}, 0.27$ to 0.65$)$ than controls, among other measures. Statistical between-study heterogeneity was observed, possibly due to variation in testing duration, device type or patient setting.

Conclusions and Relevance: Digitally captured data using wearable devices revealed that adults with dementia were less active, demonstrated increased fragmentation of their sleep-wake cycle and a loss of typical diurnal variation in circadian rhythm as compared to controls.

Keywords: technology, geriatrics, cognition, sleep, wearable

\section{INTRODUCTION AND BACKGROUND}

Dementia has been identified by the World Health Organization as a global priority for public health and social care in the twenty-first century (1). Advances in the molecular and genetic understanding of neurodegenerative disease has contributed to improved diagnostic paradigms and helped to foster a new era of personalized medicine for patients with dementia. This has coincided 
with the advancement in biological drug development for targeted therapies. These therapies have reflected the maturation in the scientific understanding of dementia that goes beyond raw measurement of cognitive performance. As a case in point a recent review of active clinical trials had shown that 14 biological treatments have targeted neuropsychiatric and behavioral symptoms as primary end-points. Challenges remain in capturing the heterogeneity of the clinical course experienced by individuals with dementia and translating these into meaningful end-points.

Technological advances using accelerometers, gyroscopes, and other motion detectors housed in mobile platforms may eventually present a cost-effective way to measure disease burden and deploy personalized treatments (2). Wearable devices that can continuously monitor physiological measures over extended periods, for example in the patient's home, provide unique information not attainable with traditional in-clinic monitoring and hold particular appeal in dementia populations (3). Advances in technology have made these devices increasingly affordable and user friendly but have been limited by methodological challenges. Specifically, their high resolution and sensitivity leaves them susceptible to noisy interference, complicated and time-consuming analytical techniques are required to derive clinically meaningful endpoints from the large amounts of data they produce, and the lack of standards has led to isolated "islands of expertise" (4).

The flexibility of wearable platforms has resulted in a variety of different uses including monitoring of gait, motion tracking, and sleep and circadian rhythm assessment (5). The ability to identify objective measurements of specific endpoints with respect to individual and group-wise subject performance, captured in realtime at various settings including at home, provides ecological validity that would otherwise be lost in laboratory settings. The main question that we had aimed to address was the potential for wearable devices to provide information on the behavioral and neuropsychiatric fluctuations inherent in the clinical course of dementia. The ability to accurately and objectively measure these fluctuations can provide researchers with viable digital surrogate end-points for use in clinical trials. We undertook a systematic review and meta-analysis to evaluate the utility of wearable technology in patients with dementia for the measurement of these neurophysiological parameters. The objective of this analysis was to systematically review studies employing wearable technology in patients with dementia by quantifying differences in digitally captured neurophysiological endpoints.

\section{LITERATURE SELECTION CRITERIA}

\section{Data Sources and Search Strategy}

Five electronic databases were searched including Cochrane, EMBASE, PubMed, PsycInfo, and IEEE. Searches were performed for Cochrane, PsycInfo, and IEEE on October 31, 2017. A PubMed search was performed on October 25, 2017, and an Embase search on October 27, 2017. A combination of Medical Subject Headings and search terms were constructed by the authors (RP, AC, and JB) in collaboration with a librarian. The Search Terms provides an outline of the search strategy for PubMed only.

\section{Search Terms. Systematic Review Search Strategy: PubMed}

1. "Dementia"[Mesh]

2. dementia

3. (frontotemporal dementia)

4. (vascular dementia)

5. (Alzheimer* disease)

6. (Parkinson* disease)

7. (lewy body)

8. Creutzfeldt-Jakob

9. 1 or 2 or 3 or 4 or 5 or 6 or 7 or 8

10. “Technology”[Mesh]

11. (wearable device)

12. (assistive technology)

13. (wearable technology)

14. on-body

15. bracelet

16. GPS

17. actigraphy

18. accelerometer

19. (galvanic skin response)

20. biosensor

21. sensor

22. gyroscope

23. watch

24. necklace

25. harness

26. strap

27. patch

28. camera

29. chip

30. (step counter)

31. pouch

32. armband

33. node

34. 10 or 11 or 12 or 13 or 14 or 15 or 16 or 17 or 18 or 19 or 20 or 21 or 22 or 23 or 24 or 25 or 26 or 27 or 28 or 29 or 30 or 31 or 32 or 33

35. 9 and 3 .

\section{Types of Studies}

We included observational studies reporting primary data in a peer-reviewed scientific journal. Studies had to include participants with a mean age $\geq 50$ years and did not include any direct intervention (i.e., drug, vitamin, supplement, exercise, cognitive, or behavioral intervention). Studies published before 1970 or translated to English were excluded. Studies that did not provide descriptive statistics for a physiological outcome were excluded. Conference abstracts, review papers, case reports, letters, opinion pieces, editorials, article comments, or corrections were excluded.

\section{Type of Exposure}

We included all-cause dementia (any dementia subtype) as our exposure (6). Exact search terms for dementia subtypes included can be found in the search strategy (Search Terms). As we included studies from 1970 onwards, diagnostic criteria 
TABLE 1 | Patient setting and diagnostic criteria for 48 observational studies testing wearable technology in participants with dementia.

\begin{tabular}{|c|c|c|}
\hline Source & Diagnostic criteria & Setting \\
\hline Aharon-Peretz et al. (7) & $\begin{array}{l}\text { DSM III-R (8)/NINCDS-ADRDA } \\
\text { (9) }\end{array}$ & Not stated \\
\hline Ahmed et al. (10) & $\begin{array}{l}\text { McKhann et al. } \\
\text { (11)/Gorno-Tempini et al. } \\
\text { (12)/Rascovsky et al. (13) }\end{array}$ & $\begin{array}{l}\text { Community (home)/ln } \\
\text { Lab }\end{array}$ \\
\hline Anderson et al. (14) & Neary criteria (15) & Community (home) \\
\hline Brown et al. (16) & Medical record diagnosis & Nursing home \\
\hline $\begin{array}{l}\text { Carvalho-Bos et al. } \\
\text { (17) }\end{array}$ & $\begin{array}{l}\text { NINCDS-ADRDA (9)/DSM-IV } \\
(18)\end{array}$ & Nursing home \\
\hline David et al. (19) & DSM-IV (18) & Out-patient clinic \\
\hline David et al. (20) & NINCDS-ADRDA (9) & Out-patient clinic \\
\hline $\begin{array}{l}\text { Eggermont and } \\
\text { Scherder (21) }\end{array}$ & Medical record diagnosis & Nursing home \\
\hline $\begin{array}{l}\text { Fetveit and Bjorvatn } \\
\text { (22) }\end{array}$ & $\begin{array}{l}\text { Clinical Dementia Rating (CDR) } \\
\text { scale (23) }\end{array}$ & Nursing home \\
\hline Fleiner et al. (24) & ICD-10 (25) & Psychiatric hospital \\
\hline Gehrman et al. (26) & $\begin{array}{l}\text { Medical record } \\
\text { diagnosis/NINCDS-ARDA (9) }\end{array}$ & Nursing home \\
\hline Ghali et al. (27) & DSM-III-R (8) & $\begin{array}{l}\text { Dementia treatment } \\
\text { Evaluation facility }\end{array}$ \\
\hline Harper et al. (28) & NINCDS-ADRDA (9) & $\begin{array}{l}\text { Hospital/clinical } \\
\text { research center }\end{array}$ \\
\hline Harper et al. (29) & NINCDS-ADRDA (9) & $\begin{array}{l}\text { Hospital/clinical } \\
\text { research center }\end{array}$ \\
\hline Hatfield et al. (30) & $\begin{array}{l}\text { DSM-IV (18)/ NINCDS-ADRDA } \\
\text { (9) }\end{array}$ & Community (home) \\
\hline $\begin{array}{l}\text { Hooghiemstra et al. } \\
\text { (31) }\end{array}$ & $\begin{array}{l}\text { DSM-V (32)/NINCDS-ADRDA } \\
\text { (9)/Neary Criteria (15)/McKeith } \\
\text { et al. (33)/Pohjasvaara et al. } \\
\text { (34) }\end{array}$ & Not stated \\
\hline $\begin{array}{l}\text { ljmker and Lamoth } \\
\text { (35) }\end{array}$ & $\begin{array}{l}\text { Clinician/ medical record } \\
\text { diagnosis }\end{array}$ & In laboratory \\
\hline Iwata et al. (36) & $\begin{array}{l}\text { DSM-IV (18)/NINCDS-ARDA } \\
\text { (9) }\end{array}$ & Not stated \\
\hline James et al. (37) & NINCDS-ARDA (9) & Community (home) \\
\hline Kodama et al. (38) & DSM-III-R (8) & Community (home) \\
\hline König et al. (39) & $\begin{array}{l}\text { International working group-2 } \\
\text { criteria (IWG-2) (40) }\end{array}$ & Memory clinic \\
\hline Kuhlmei et al. (41) & $\begin{array}{l}\text { NINCDS-ADRDA } \\
\text { (9)/NINDS-AIREN (42) }\end{array}$ & Not stated \\
\hline La Morgia et al. (43) & NINCDS-ADRDA (9) & Not stated \\
\hline Lamoth et al. (44) & Alzheimer's association criteria & Clinic/hospital \\
\hline Landolt et al. (45) & Autopsy or biopsy confirmation & $\begin{array}{l}\text { Hospital/ nursing } \\
\text { home }\end{array}$ \\
\hline Lee et al. (46) & NINCDS-ADRDA (9) & Not stated \\
\hline Leger et al. (47) & $\begin{array}{l}\text { DSM-V (32)/ NINCDS-ADRDA } \\
\text { (9)/MMSE } \leq 25 \text { and } \geq 15 \\
\text { (48)/CDR (Score of } 0.5,1 \text {, or } 2) \\
\text { (23) }\end{array}$ & Out-patient clinic \\
\hline McCurry et al. (49) & $\begin{array}{l}\text { Family physician/medical } \\
\text { record diagnosis }\end{array}$ & Community (home) \\
\hline Merrilees et al. (50) & $\begin{array}{l}\text { Neary criteria for } \\
\text { frontotemporal lobar } \\
\text { degeneration (51) }\end{array}$ & Community (home) \\
\hline Most et al. (52) & NINCDS- ADRDA (9) & Not stated \\
\hline
\end{tabular}

(Continued)
TABLE 1 | Continued

\begin{tabular}{|c|c|c|}
\hline Source & Diagnostic criteria & Setting \\
\hline Moyle et al. (53) & Medical record diagnosis & Long-term care facility \\
\hline Mulin et al. (54) & NINCDS- ADRDA (9) & Community (home) \\
\hline Murphy et al. (55) & MMSE < 23 (48) & Nursing home \\
\hline Olsen et al. (56) & $\begin{array}{l}\text { Medical record diagnosis or } \\
\text { MMSE }<25 \text { (48) }\end{array}$ & $\begin{array}{l}\text { Nursing } \\
\text { home/community } \\
\text { (home) }\end{array}$ \\
\hline Paavilainen et al. (57) & $\begin{array}{l}\mathrm{CDR}>0.5(23) / \mathrm{MMSE}<20 \\
(48)\end{array}$ & Nursing home \\
\hline Pollak and Stokes (58) & $\begin{array}{l}\text { Mattis dementia rating scale } \\
\text { total score }<123(59) \text { Mattis } \\
\text { dementia rating scale memory } \\
\text { score }<19(59)\end{array}$ & Community (home) \\
\hline $\begin{array}{l}\text { Rindlisbacher and } \\
\text { Hopkins (60) }\end{array}$ & DSM-III-R (8) & Hospital \\
\hline Schwenk et al. (61) & $\begin{array}{l}\text { NINCDS-ADRDA } \\
\text { (9)/NINDS-AIREN (42) }\end{array}$ & Community (home) \\
\hline Valembois et al. (62) & DSM-IV (18) & Hospital \\
\hline van Alphen et al. (63) & Medical record diagnosis & $\begin{array}{l}\text { Community } \\
\text { (home)/nursing home }\end{array}$ \\
\hline $\begin{array}{l}\text { van Someren et al. } \\
\text { (64) }\end{array}$ & $\begin{array}{l}\text { DSM-III-R (8)/NINCDS-ADRDA } \\
\text { (9) }\end{array}$ & $\begin{array}{l}\text { Community } \\
\text { (home)/nursing home }\end{array}$ \\
\hline Varma and Watts (65) & NINCDS-ADRDA (9) & Community (home) \\
\hline Viegas et al. (66) & DSM-IV (18)/MMSE $\leq 24$ (48) & Nursing home \\
\hline Volicer et al. (67) & $\begin{array}{l}\text { NINCDS-ADRDA (9)/DSM-III-R } \\
\text { (8) }\end{array}$ & Hospital \\
\hline Wams et al. (68) & NINCDS-ADRDA (9) & Community (home) \\
\hline Weissova et al. (69) & NINCDS-ADRDA (9) & Community (home) \\
\hline Wirz-Justice et al. (70) & DSM -IV (18) & Hospital \\
\hline Yesavage et al. (71) & NINCDS-ADRDA (9) & Community (home) \\
\hline
\end{tabular}

for the diagnosis of dementia differed between studies and is summarized for each study in Table 1.

\section{Types of Outcome Measures}

We included studies which provided physiological data as measured by wearable technology. Wearable technology was defined as a non-implantable, body-fixed sensor technology designed to monitor for $>24 \mathrm{~h}$ and to not interfere with the wearer's normal activity $(5,72)$. By this definition, studies using finger-based pulse oximeters, blood pressure monitors, galvanic skin response sensors, functional near-infrared spectroscopy (fNIRS), and electroencephalograms (EEG) were excluded. Where studies included measurement devices other than a wearable device, only data from the wearable device was included in the final analysis.

\section{Methods for Literature Secondary Screening First Selection: Abstract Screening}

Two authors (RP and AC) independently screened each record by title and abstract according to eligibility criteria. Eighteen thousand four hundred fifty-six abstracts were included in the initial screening process. There were 525 disagreements in abstract selection between the two reviewers. Conflicts were 


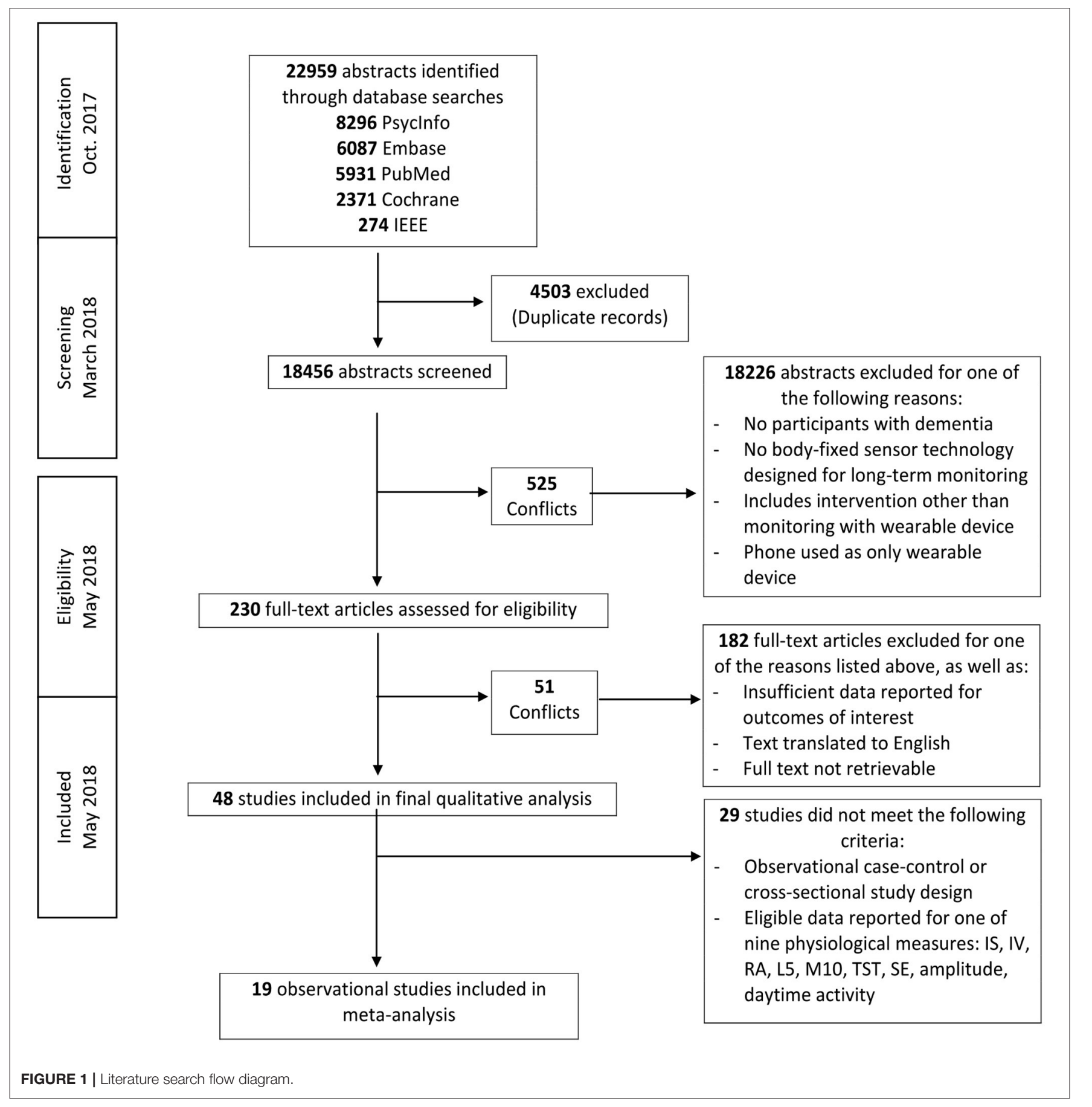

resolved by two additional authors (NS and JB) using the inclusion and exclusion criteria and definitions outlined in Figure 1.

\section{Final Selection}

Two hundred thirty articles were eligible for full text review. Two authors (RP and AC) independently determined eligibility of each article for inclusion. In cases of disagreement or conflict, senior authors (NS, JB, and KT) determined whether the study met eligibility criteria. Forty-eight articles were included in the final systematic review.

\section{Data Collection}

Data was extracted by three authors (JB, RP, and AC). Information extracted from each publication is provided in Table 6. To assess the methodological quality of included studies, we used the checklist provided by Downs and Black (73). A total quality score is provided for each study in Table 6 (maximum score $=32$ ). 


\section{Statistical Analyses}

Data analysis was performed using Stata/SE (StataCorp LP, Texas, Version 15). The age and number of included participants per study, as well as general study results are provided in Table 6. Initial synthesis of qualitative data revealed a number of common endpoints reported consistently by authors. Subsequent meta-analyses included only observational case-control or crosssectional studies that presented data for these commonly reported endpoints (Table 7).

Meta-analyses were conducted using the standardized mean difference (Hedges' g). Hedges' g values $\leq 0.20,>0.20$ but $<0.80$, and $\geq 0.80$ were considered small, moderate, or large, respectively between controls and participants with dementia (74). For each single or combined effect size, a positive value indicated a higher mean value of that variable in participants with dementia than in healthy volunteers. Some publications contained two subgroups of dementia participants. A fixed effects meta-analysis was performed on the dementia subgroups within each of these studies to compute a composite effect size and variance (75). This composite effect was used in the across-study random effects analysis.

Across-study heterogeneity was investigated using the Cochran's Q-test and $I^{2}$ statistic. Cochran's Q test was performed using the weighted method of moments method (75). Cochran's $\mathrm{Q}$ statistic was considered significant at $p<0.10 . I^{2}$-values of 25 , 50 , and $75 \%$ were considered indicative of low, moderate, and high heterogeneity, respectively (76).

For each analysis, a funnel plot of standardized mean differences was constructed, and the risk of publication bias evaluated through funnel plot asymmetry and Egger tests. We acknowledge that many other factors including heterogeneity, differences in methodological quality, and selective reporting may produce funnel plot asymmetry (77).

The influence of each study on a meta-analysis estimate was investigated through influence analysis, where each individual study is omitted in turn and the meta-analysis re-estimated using a random effects model. For publications that included more than one subgroup of participants with dementia, the largest subgroup was included in the influence analysis.

There was no funding source for this study and the corresponding author had full access to all of the data in the study and had final responsibility for the decision to submit for publication.

\section{RESULTS}

\section{Systematic Review}

Five database searches resulted in 18,456 retrieved abstracts after removal of duplicates (Figure 1). Two hundred thirty of these publications qualified for full-text screening after examination by title and abstract. Forty-eight studies qualified for inclusion in the final qualitative analysis and 19 of these publications qualified for inclusion in one or multiple meta-analyses (Dementia: $n=617$, Control: $n=406$ ) (Table 7).

Nineteen studies (39\%) enrolled participants only with adrelated diagnoses. Table 2 describes the technical specifications of devices used in individual studies. Thirty-four studies (70\%) tested participants using a wrist-worn actigraph. The average assigned duration of wear was 8.26 days (range: 6 min-28 days). Forty (83\%) studies used accelerometry as the main measurement of activity. One study used an accelerometer with a gyroscope, while one further study used an accelerometer, gyroscope and magnetometer. Six studies (12\%) used activity monitors which did not state the type of measurement modality.

\section{Daily Activity}

Of the 48 included studies, 23 (47\%) groups reported outcome data on daily activity counts as measured by actigraphy. Qualitative analysis showed that activity counts were presented in a number of different ways (Table 3). Significant associations of activity counts with other measures, or differences in activity between individuals with dementia and control groups, were reported for the measures of daily activity (eight groups, $34 \%$ ), peak daily activity (two groups, $8 \%$ ), mean activity counts (five groups, 21\%), daytime activity (five groups, $21 \%$ ), night time activity (one group, 4\%), number of immobile hours (one group, 4\%), and activity patterns (three groups, $13 \%)$. Quantitative analysis demonstrated that participants with dementia had a significantly lower mean daytime activity counts compared to controls (mean difference, $-1.60 ; 95 \%$ CI, -2.66 to -0.55$)$ (Dementia: $n=210$, Control: $n=136$ ) (Figure 2I).

\section{Wearable Actigraphy for Sleep Derived Measures}

Of the 48 included studies, 31 (64\%) groups reported outcome data on sleep characteristics as measured by actigraphy (Table 4). Wake after sleep onset (WASO) was reported by 11 groups: six (55\%) reported a significant association or difference in dementia subjects. Total sleep time (TST) was reported by 16 groups: nine (56\%) reported a significant association or difference in dementia subjects. Sleep efficiency (SE) was reported by 12 groups: five $(42 \%)$ reported a significant association or difference in dementia subjects. Participants with dementia had statistically significant lower mean sleep efficiency than controls (mean difference, -0.52 ; $95 \% \mathrm{CI},-0.89$ to -0.16 ) (Dementia: $n=193$, Control: $n=171$ ) (Figure 2G), and no significant difference in mean total sleep time (mean difference, $0.46 ; 95 \% \mathrm{CI},-0.21$ to 1.12 ) (Dementia: $n=291$, Control: $n=212$ ) (Figure 2F).

\section{Non-parametric Measurements of Circadian Rhythm Using Wearable Devices}

Sixteen (33\%) of 48 studies reported non-parametric measurements of circadian rhythm (Table 4). Qualitative analysis revealed that intradaily variability (IV) was reported by 13 groups: eight (61\%) reported an association or difference in dementia groups. Interdaily stability (IS) was reported by 14 groups: nine (64\%) reported an association or difference in dementia subjects. Relative amplitude (RA) was reported by 12 groups: seven (58\%) reported an association or difference in dementia subjects. Activity of most active $10 \mathrm{~h}$ (M10) was reported by nine groups: seven (77\%) reported an association or difference in dementia subjects. Activity of least active 5 h (L5) 
TABLE 2 | Technical specifications of wearable devices used in individual studies.

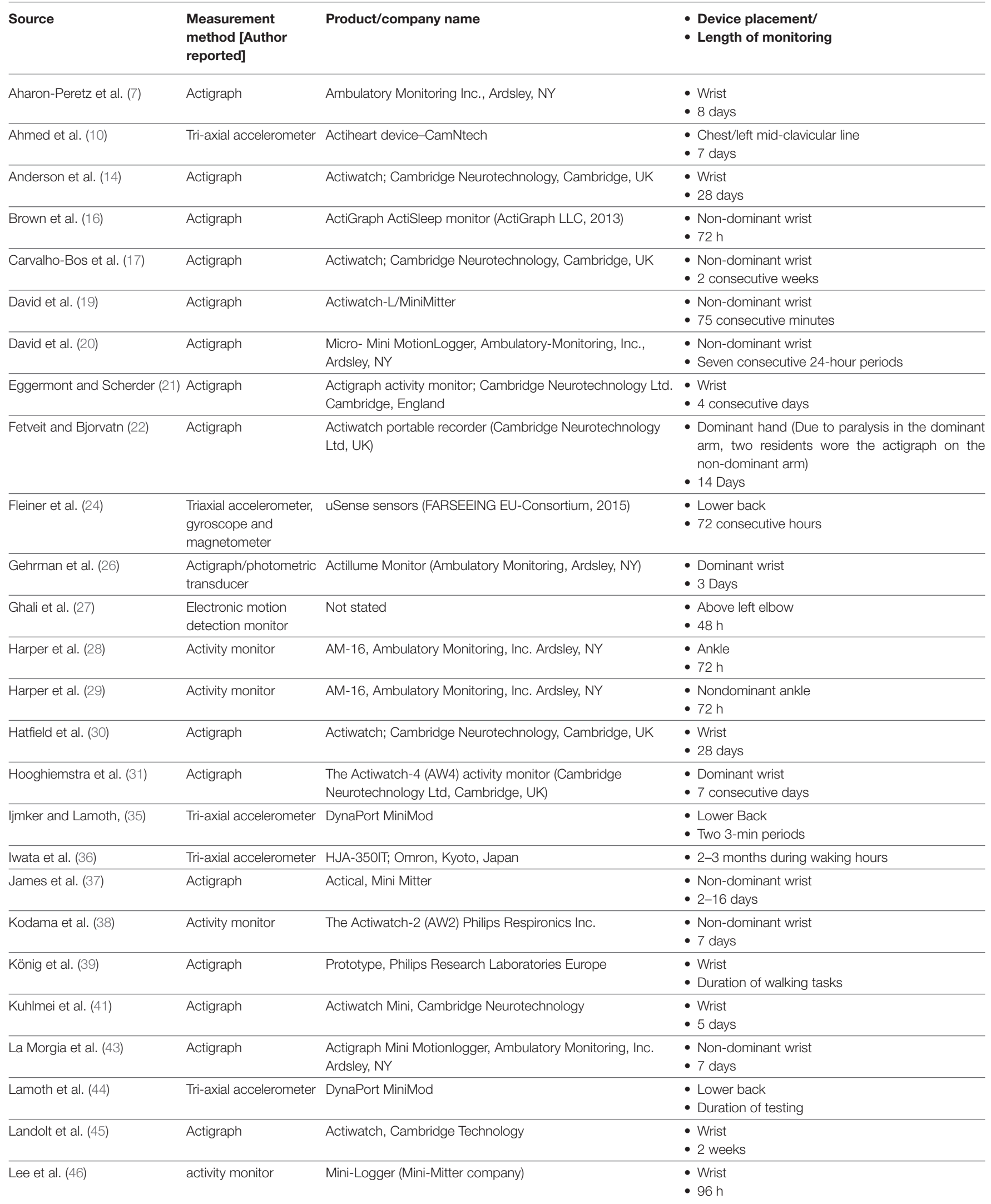


TABLE 2 | Continued

\begin{tabular}{|c|c|c|c|}
\hline Source & $\begin{array}{l}\text { Measurement } \\
\text { method [Author } \\
\text { reported] }\end{array}$ & Product/company name & $\begin{array}{l}\text { - Device placement/ } \\
\text { - Length of monitoring }\end{array}$ \\
\hline Leger et al. (47) & Actigraph & Motionwatch 8 (MW8, Camntech, Cambridge, UK) & $\begin{array}{l}\text { - Non-dominant wrist } \\
\text { - } 14 \text { days }\end{array}$ \\
\hline McCurry et al. (49) & Actigraph & $\begin{array}{l}\text { Actillume wrist-movement recorder (Ambulatory Monitoring, } \\
\text { Inc., Ardsley, NY) }\end{array}$ & $\begin{array}{l}\text { - Wrist } \\
\text { - } 7 \text { days }\end{array}$ \\
\hline Merrilees et al. (50) & Actigraph & MiniMitter Actiwatch monitors (AW-64) & $\begin{array}{l}\text { - Non-dominant wrist } \\
\text { - } 2 \text { weeks }\end{array}$ \\
\hline Most et al. (52) & Actigraph & Actiwatch; Cambridge Neurotechnology, Cambridge, UK & $\begin{array}{l}\text { - Non-dominant wrist } \\
\text { - } 2 \text { weeks }\end{array}$ \\
\hline Moyle et al. (53) & Tri-axial accelerometer & $\begin{array}{l}\text { SenseWear }{ }^{\circledR} \text { Professional } 8.0 \text { activity armband (Temple } \\
\text { Healthcare, BodyMedia, Inc) }\end{array}$ & $\begin{array}{l}\text { - Upper non-dominant arm } \\
\text { - Monday to Saturday }\end{array}$ \\
\hline Mulin et al. (54) & Actigraph & $\begin{array}{l}\text { Micro- Mini MotionLogger, Ambulatory-Monitoring, Inc., } \\
\text { Ardsley, NY }\end{array}$ & $\begin{array}{l}\text { - Non-dominant wrist } \\
\text { - } 7 \text { days }\end{array}$ \\
\hline Murphy et al. (55) & Tri-axial accelerometer & Sensewear Armband, Body Media & $\begin{array}{l}\text { - Upper left arm } \\
\text { - } 7 \text { days }\end{array}$ \\
\hline Olsen et al. (56) & Actigraph & ActiSleep+, Actigraph, Pensacola, USA & $\begin{array}{l}\text { - Left wrist } \\
\text { - } 7 \text { days }\end{array}$ \\
\hline Paavilainen et al. (57) & $\begin{array}{l}\text { Telemonitoring and } \\
\text { actigraphy system }\end{array}$ & Information Security Technology (IST) Vivago system & $\begin{array}{l}\text { - Wrist } \\
\text { - 9-113 days }\end{array}$ \\
\hline Pollak and Stokes, (58) & Activity Monitor & MiniMotionlogger recorder (Ambulatory Monitoring, Inc.) & $\begin{array}{l}\text { - Non-dominant wrist } \\
\text { - } 9 \text { days }\end{array}$ \\
\hline $\begin{array}{l}\text { Rindlisbacher and Hopkins, } \\
\text { (60) }\end{array}$ & $\begin{array}{l}\text { Ambulatory monitoring } \\
\text { device }\end{array}$ & Not stated & $\begin{array}{l}\text { - Above left elbow (in shirt) } \\
\text { - Four consecutive days }\end{array}$ \\
\hline Schwenk et al. (61) & $\begin{array}{l}\text { Accelerometer/ } \\
\text { gyroscope }\end{array}$ & Physilog (BioAGM, CH) & $\begin{array}{l}\text { - Chest } \\
\text { - } 24 \mathrm{~h}\end{array}$ \\
\hline Valembois et al. (62) & Actigraph & Vivago, Vivago Oy, Espoo, Finland & $\begin{array}{l}\text { - Non-dominant wrist } \\
\text { - } 10 \text { days }\end{array}$ \\
\hline Van Alphen et al. (63) & Tri-axial accelerometer & $\begin{array}{l}\text { The Actiwatch-4 (AW4) activity monitor (Cambridge } \\
\text { Neurotechnology Ltd, Cambridge, UK) }\end{array}$ & $\begin{array}{l}\text { - Dominant wrist } \\
\text { - } 6 \text { days }\end{array}$ \\
\hline van Someren et al. (64) & Actigraph & Not stated & $\begin{array}{l}\text { - Wrist } \\
\text { - } 155 \mathrm{~h}\end{array}$ \\
\hline Varma and Watts, (65) & Actigraph & Actigraph GT3X+ & $\begin{array}{l}\text { - Dominant hip } \\
\text { - } 7 \text { days }\end{array}$ \\
\hline Viegas et al. (66) & Actigraph & $\begin{array}{l}\text { Basic Mini-Motionlogger Actigraph, Ambulatory Monitoring, } \\
\text { Inc. }\end{array}$ & $\begin{array}{l}\text { - Wrist } \\
\text { - Five 24-h periods }\end{array}$ \\
\hline Volicer et al. (67) & Activity monitor & AM-16 Ambulatory Monitoring, Ardsley NY & $\begin{array}{l}\text { - Waist } \\
\text { - } 72 \mathrm{~h}\end{array}$ \\
\hline Wams et al. (68) & Actigraph & Actiwatch 7, CamNTech Ltd. & $\begin{array}{l}\text { - Non-dominant wrist } \\
\text { - } 3 \text { weeks }\end{array}$ \\
\hline Weissova et al. (69) & Actigraph & Actiwatch, AW4 model, Cambridge Neurotechnology Ltd. & $\begin{array}{l}\text { - Non-dominant wrist } \\
\text { - } 21 \text { days }\end{array}$ \\
\hline Wirz-Justice et al. (70) & Actigraph with luxmeter & r Actiwatch-L, Cambridge Neurotechnologies & $\begin{array}{l}\text { - Non-dominant wrist } \\
\text { - 10-26 days }\end{array}$ \\
\hline Yesavage et al. (71) & Actigraph & Ambulatory Monitoring Systems, Inc. Ardsley, NY & $\begin{array}{l}\text { - Non-dominant wrist } \\
\text { - } 6 \text { days }\end{array}$ \\
\hline
\end{tabular}

was reported by eight groups: four (50\%) reported an association or difference in dementia groups.

Participants with dementia had significantly lower mean values than controls on IS (mean difference, -0.47 ; 95\% CI, -0.81 to $-0 \cdot 14$ ) (Dementia: $n=298$, Control: $n=180$ ) (Figure 2A), RA (mean difference, -0.53 ; $95 \% \mathrm{CI},-0.76$ to -0.30) (Dementia: $n=237$, Control: $n=152$ ) (Figure 2C), and M10 (mean difference, $-0.61 ; 95 \% \mathrm{CI},-1.16$ to -0.06 ) (Dementia: $n=184$, Control: $n=103$ ) (Figure 2E) outcomes. Participants with dementia had statistically significantly higher mean values than controls on IV (mean difference, $0.46 ; 95 \% \mathrm{CI}$, 0.27-0.65) (Dementia: $n=245$, Control: $n=171$ ) (Figure 2B) and L5 (mean difference, 0.38; 95\% CI, 0.15-0.61) (Dementia: $n$ $=184$, Control: $n=103$ ) (Figure 2D) outcomes. 


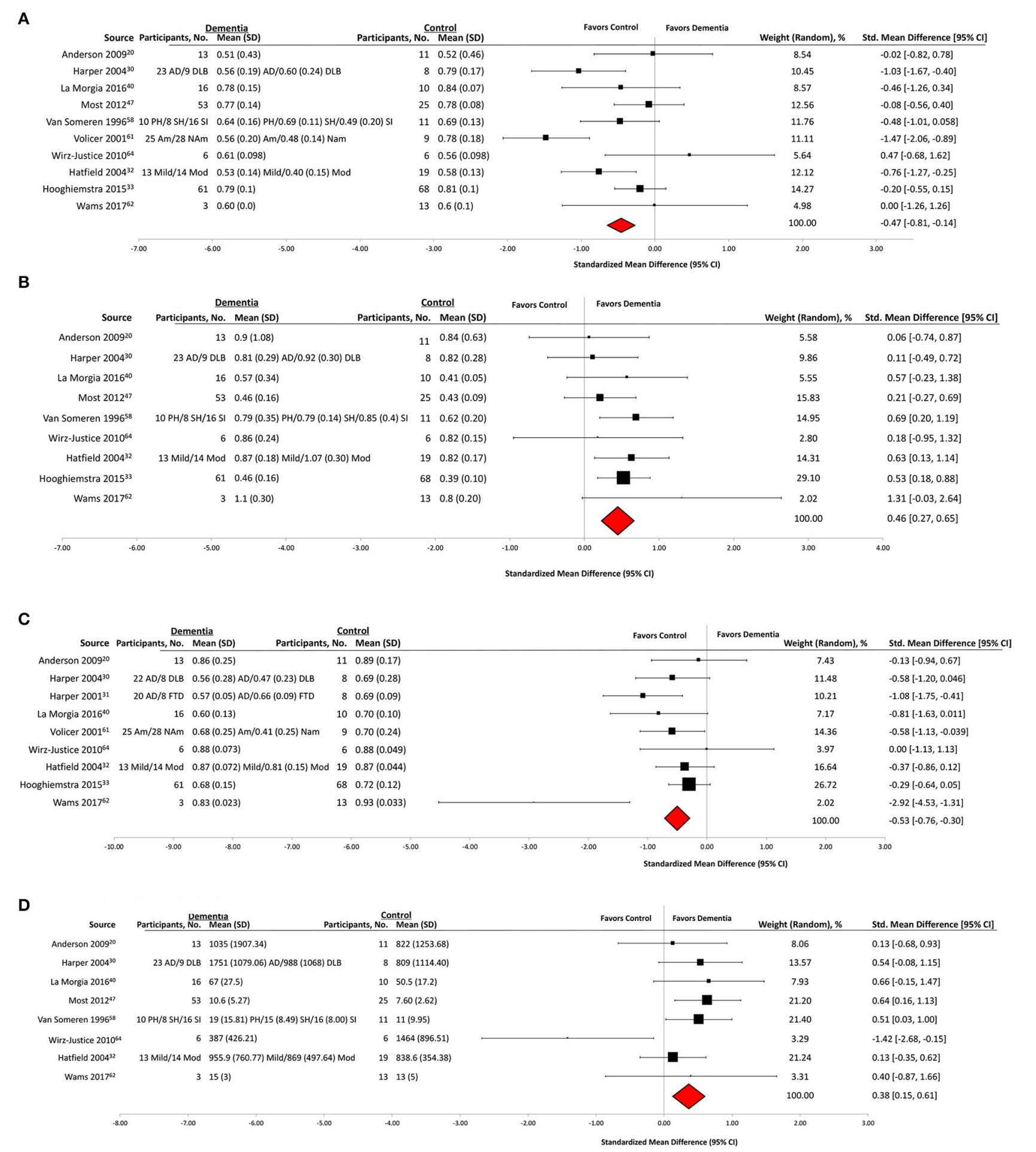

FIGURE 2 | Continued 


\section{E}

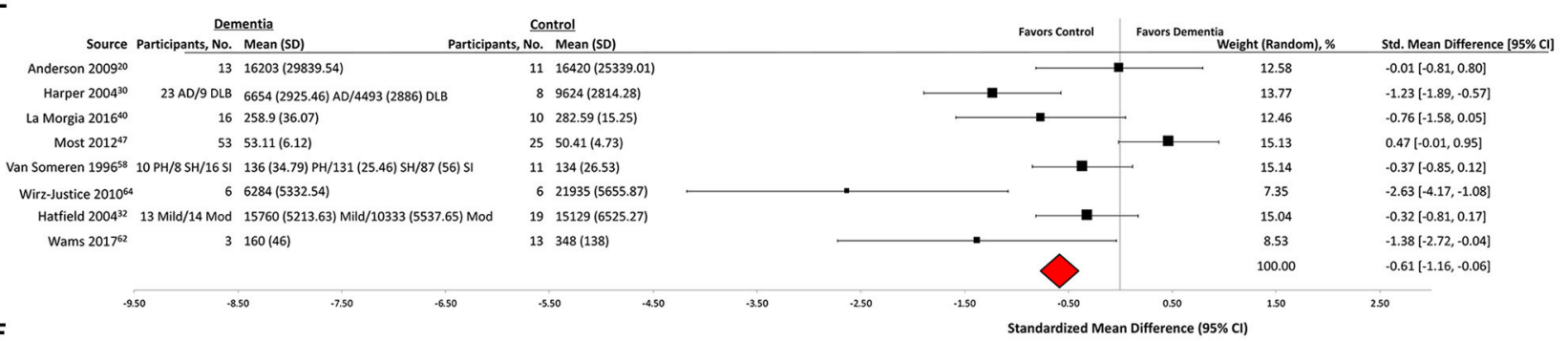

$\mathbf{F}$ \begin{tabular}{llll}
\multicolumn{1}{c}{ Dementia } & Control & Favors Control & Favors Dementia
\end{tabular} Aharon-Peretz 199118 15 DAT/10 MID 8.57 (1.92) DAT/8.74 (1.37) MID $11 \quad 7.24(1.02)$

$$
\text { Lee 2004 } \quad 7 \quad 3.34(0.31)
$$

Hooghiemstra $2015^{33}$

Brown $2015^{21}$

$\begin{array}{lll}61 & 6.22(1.37)\end{array}$

Valembois $2015^{56}$

Wams $2017^{62}$

$$
2212.07 \text { (5.59) }
$$

120495 (326)

Most $2012^{47}$

$38.45(0.58)$

$53396.6(67.1)$
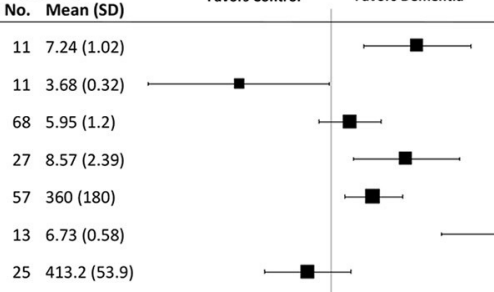

Weight (Random), \% Std. Mean Difference [95\% Cl]

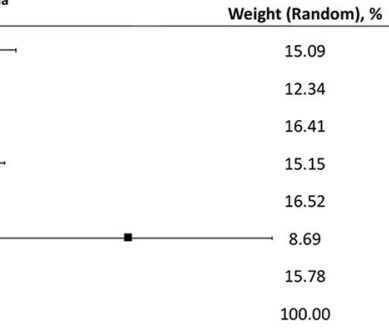
$0.95[0.36,1.55]$

$-1.02[-2.03,-0.02]$

$0.21[-0.14,0.55]]$

$0.83[0.25,1.42]$

$0.47[0.15,0.79]$

$2.80[1.22,4.39]$

$-0.26[-0.74,0.22]$

$0.46[-0.21,1.12]$

$\mathbf{G}$

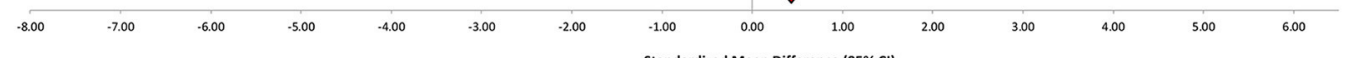

\section{Dementia $\quad$ Control}

Standardized Mean Difference $(95 \% \mathrm{Cl})$

$\begin{array}{rrlll}\text { Aharon-Peretz 199118 } & \text { 15 DAT/10 MID } & 82.85(8.01) \text { DAT/61.66 (23.74) MID } & 11 & 81.89(5.07) \\ \text { La Morgia 2016 } & 16 & 75.4(9.58) & 10 & 90.2(6.10) \\ \text { Lee 2004 } & 7 & 52.17(3.46) & 11 & 56.92(4.32) \\ \text { Most 2012 } & 53 & 72.9(10.1) & 25 & 79.8(5.8) \\ \text { Wirz-Justice 2010 } & 6 & 87(7.10) & 6 & 88.6(5.63) \\ \text { Brown 201521 } & 22 & 91.21(8.45) & 27 & 91.33(6.05) \\ \text { Hooghiemstra 2015 } & 61 & 71.9(15.1) & 68 & 74.1(11.6) \\ \text { Wams 2017 } & 3 & 89.3(1.3) & 13 & 90.4(3.8)\end{array}$

H

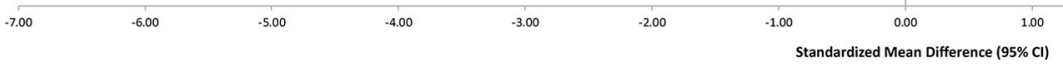

Weight (Random), \% Std. Mean Difference [95\% Cl]

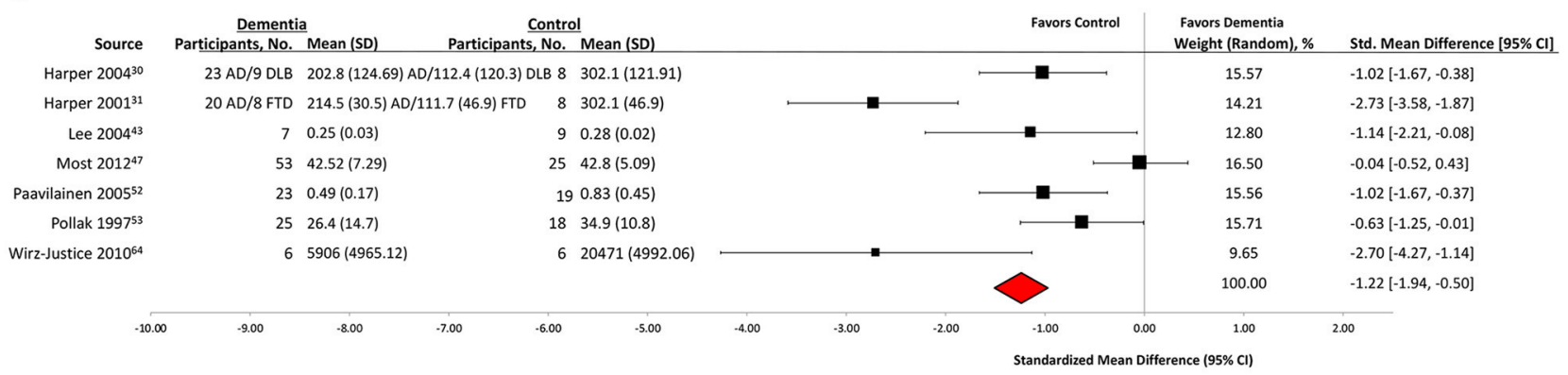

\begin{tabular}{|c|c|c|c|c|}
\hline Study & $\begin{array}{l}\text { Dem } \\
\text { Total }\end{array}$ & $\frac{\text { mentia }}{\text { Mean (SD) }}$ & & $\frac{\text { ntrol }}{\text { Mean (SD) }}$ \\
\hline Harper 2004 & $23 \mathrm{AD} / 9 \mathrm{DLB}$ & $403.4(179.36) \mathrm{AD} / 263.6(178.2) \mathrm{DLB}$ & $B 8$ & $560(178.19)$ \\
\hline Paavilainen 2005 & 23 & $1.36(0.46)$ & 19 & $1.69(0.57)$ \\
\hline Pollak 1997 & 25 & $60.5(27.8)$ & 18 & $80.4(27.1)$ \\
\hline Varma 2017 & 39 & $378119(32004.3)$ & 53 & $514326.3(28624.64)$ \\
\hline Volicer 2001 & $25 \mathrm{Am} / 28 \mathrm{NAm}$ & 348 (206) Am/277 (228) NAm & 9 & $608(206)$ \\
\hline Wirz-Justice 2010 & 6 & $38.3(11.76)$ & 6 & $135.6(66.87)$ \\
\hline Kuhlmei 2013 & $12 \mathrm{NAp} / 20 \mathrm{Ap}$ & $43.65(13.79) \mathrm{NAp} / 38.76$ (15.1) Ap & 23 & $57.46(16.77)$ \\
\hline
\end{tabular}

1

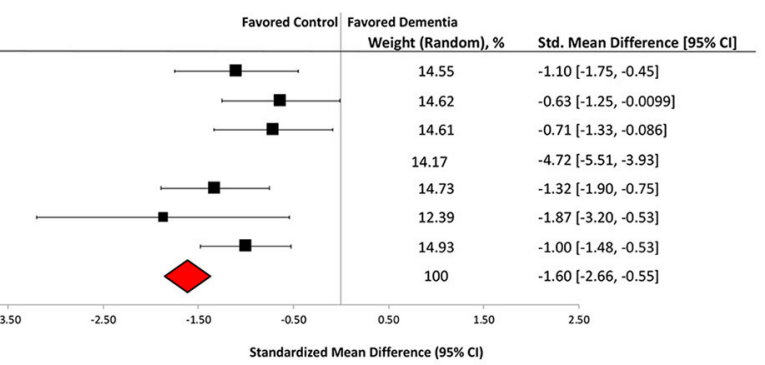

FIGURE 2 | Actigraphy outcomes in observational case control studies of wearable technology. (A) Interdaily stability, (B) interdaily variability, (C) relative amplitude, (D) activity of least active $5 \mathrm{~h}$, (E) Activity of most active $10 \mathrm{~h}$, (F) total sleep time, (G) sleep efficiency, (H) amplitude, and (I) daytime activity. 
TABLE 3 | Specific outcome measures of daily activity reported by included studies.

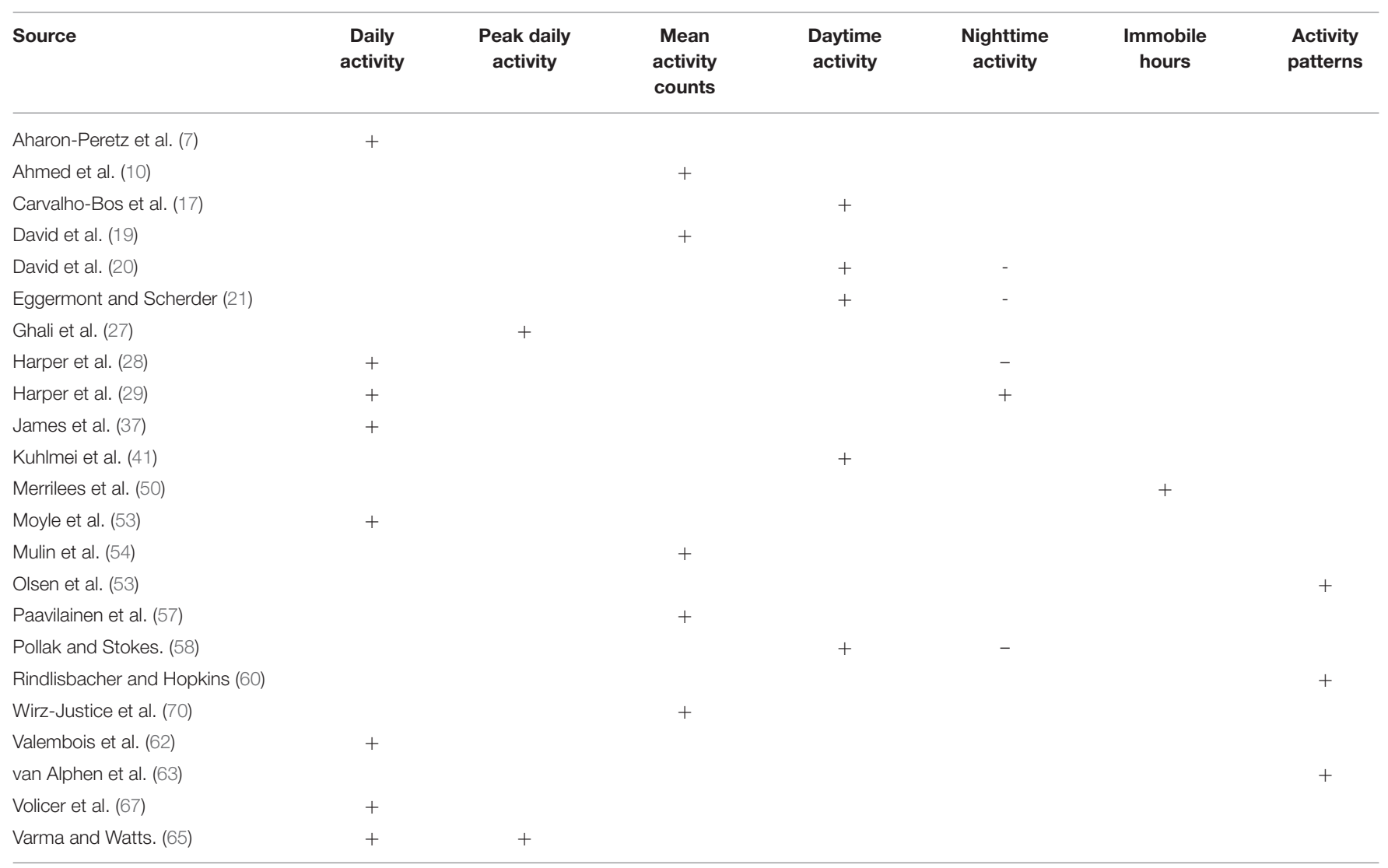

+ indicates a significant association or difference reported.

-indicates no significant association or difference reported.

\section{Cosinor Analysis of Circadian Rhythm Using Wearable Devices}

Nine (19\%) out of the 48 groups reported a cosinor analysis of circadian rhythm. Qualitative analysis (Table 4) showed that midline estimating statistic of rhythm (mesor) was reported by five groups: two (40\%) reported a significant association or difference in dementia subjects. Amplitude of the cosinor wave was reported by 10 groups: five (50\%) reported an association or difference in dementia subjects. Acrophase was reported by eight groups: two (25\%) reported an association or difference in dementia subjects. Quantitative analysis was only performed on the amplitude of the cosinor wave. It revealed that subjects with dementia had a significantly lower mean amplitude than controls (mean difference, -1.22 ; 95\% CI, -1.94 to -0.50 ) (Dementia: $n=174$, Control: $n=93$ ) (Figure $2 \mathbf{H}$ ).

\section{Wearable Actigraphy for Gait Derived Measures}

Of the 48 included studies six (12\%) groups reported outcome data on actigraphy to measure posture and gait characteristics (Table 5). Qualitative analysis showed that all six (100\%) reported an association or difference in dementia subjects. These studies each reported a different measure of gait or walking activity, and thus a meta-analysis was not possible.

\section{Risk of Bias Within Studies}

Average rating of methodological quality of included studies was 15.54 points $(S D=1 \cdot 47)$. The median and mode were both 16 points, with a range of $12-18$ (Table 6).

\section{Meta-Analysis and Heterogeneity}

Low between study heterogeneity $\left(I^{2}<50 \%\right)$ was observed for analyses of IV, RA, and L5 variables (Table 7). Moderate to high between study heterogeneity $\left(I^{2}>50 \%\right)$ was observed for analyses of IS, TST, amplitude, M10, SE, and daytime activity. Meta-regression or subgroup analyses were performed for all actigraphy measures with a moderate to high heterogeneity $\left(I^{2}\right.$ $>50 \%$ ) which included IS, TST, Amplitude, M10, SE, and daytime activity. Type of dementia, mean age, study design and quality score were all investigated as explanatory variables. Subgroup analyses indicate that effect estimates vary markedly between dementia subtypes for variables M10 and SE, suggesting differences in dementia type between studies may account for some of the heterogeneity observed in meta-analyses of M10 and SE measurements. 
TABLE 4 | Specific outcome measures of sleep and circadian rhythm reported by included studies.

\begin{tabular}{|c|c|c|c|c|c|c|c|c|c|c|c|}
\hline Source & WASO & TST & SE & IV & IS & RA & M10 & L5 & Mesor & Acrophase & Amplitude \\
\hline Aharon-Peretz et al. (7) & & - & + & & & & & & & & \\
\hline Anderson et al. (14) & & & & - & - & - & - & - & & & \\
\hline Brown et al. (16) & & + & - & & & & & & & & \\
\hline Carvalho-Bos et al. (17) & & & & + & + & + & + & + & & & \\
\hline Eggermont and Scherder (21) & & & & + & + & + & & & & & \\
\hline Fetveit and Bjorvatn (22) & + & + & - & & & & & & - & - & - \\
\hline Gehrman et al. (26) & & & & & & & & & - & - & - \\
\hline Harper et al. (28) & & & & - & + & - & + & + & + & + & + \\
\hline Harper et al. (29) & & & & + & + & - & + & + & + & & + \\
\hline Hatfield et al. (30) & & & & + & + & + & + & - & & & \\
\hline Hooghiemstra et al. (31) & + & + & - & + & - & + & & & & & \\
\hline Kodama et al. (38) & & & & + & + & + & & & & & \\
\hline La Morgia et al. (43) & & & + & - & - & + & + & - & & & \\
\hline Landolt et al. (45) & & + & & & & & & & & & \\
\hline Lee et al. (46) & - & - & - & & & & & & & - & - \\
\hline Leger et al. (47) & - & + & + & - & - & & & & & & \\
\hline McCurry et al. (49) & & - & & & & & & & & & \\
\hline Most et al. (52) & + & - & + & + & + & - & - & - & & & \\
\hline Mulin et al. (50) & + & - & & & & & & & & & \\
\hline Murphy et al. (55) & & + & & & & & & & & & \\
\hline Olsen et al. (56) & - & - & & & & & & & & & \\
\hline Paavilainen et al. (57) & & & & & & & & & & - & + \\
\hline Pollak and Stokes (58) & & & & & & & & & & - & + \\
\hline van Someren et al. (64) & & & & + & + & + & + & & & & \\
\hline Viegas et al. (66) & + & + & & & & & & & & & \\
\hline Volicer et al. (67) & & & & & + & & & & & + & - \\
\hline Wams et al. (68) & - & + & - & & & & & & & & \\
\hline Weissova et al. (69) & - & + & - & & & & & & & & \\
\hline Wirz-Justice et al. (70) & - & & - & - & - & - & + & + & & & + \\
\hline Yesavage et al. (71) & + & - & + & & & & & & - & - & - \\
\hline
\end{tabular}

+indicates a significant association or difference reported.

-indicates no significant association or difference reported.

IS, Interdaily stability; IV, Intradaily variability; L5, Activity of least active 5 h; M10, Activity of most active 10 h; RA, Relative amplitude; SE, Sleep efficiency; TST, Total sleep time; WASO, Wake after sleep onset.

TABLE 5 | Specific outcome measures of gait and walking activity reported by included studies.

\begin{tabular}{|c|c|c|c|c|c|c|c|c|}
\hline Source & Gait & Gait speed & $\begin{array}{l}\text { Walking } \\
\text { speed }\end{array}$ & Cadence & $\begin{array}{c}\text { Step } \\
\text { variance }\end{array}$ & Dual tasking & $\begin{array}{l}\text { Walking } \\
\text { duration }\end{array}$ & $\begin{array}{r}\text { Physical } \\
\text { activity }\end{array}$ \\
\hline $\begin{array}{l}\text { Aharon-Peretz } \\
\text { et al. ( } 7 \text { ) }\end{array}$ & + & & & & & & & \\
\hline Harper et al. (29) & & + & & & & & & \\
\hline Iwata et al. (36) & & & + & - & - & & & \\
\hline $\begin{array}{l}\text { La Morgia et al. } \\
\text { (43) }\end{array}$ & & & & & & + & & \\
\hline $\begin{array}{l}\text { Van Alphen et al. } \\
\text { (63) }\end{array}$ & & & & & & & + & \\
\hline Volicer et al. (67) & & & & & & & & + \\
\hline
\end{tabular}

+ , Indicates a significant association or difference reported.

-, Indicates no significant association or difference reported. 
TABLE 6 | Characteristics and major findings of included studies.

\begin{tabular}{|c|c|c|c|c|c|}
\hline Source & Study design & Participants $(n)$ & $\begin{array}{l}\text { Age, mean (SD } \\
\text { or range), y }\end{array}$ & Major findings & $\begin{array}{l}\text { Quality } \\
\text { score }^{\dagger}\end{array}$ \\
\hline Aharon-Peretz et al. $(7)^{\ddagger}$ & $\begin{array}{l}\text { Prospective } \\
\text { Case control }\end{array}$ & $\begin{array}{l}\text { MID (10) } \\
\text { AD (15) } \\
\text { Control (11) }\end{array}$ & $\begin{array}{l}\text { MID } 75.9(8.2) \\
\text { AD } 72.8(6.3) \\
\text { Control } \\
69.0(3.4)\end{array}$ & $\begin{array}{l}\text { Groups with dementia demonstrated significant differences in } \\
\text { sleep efficiency and total daily activity but not total sleep time. }\end{array}$ & 13 \\
\hline Ahmed et al. (10) & $\begin{array}{l}\text { Prospective } \\
\text { case control }\end{array}$ & $\begin{array}{l}\text { FTD (19) } \\
\text { AD (13) } \\
\text { Control (16) }\end{array}$ & Not Stated & $\begin{array}{l}\text { Decreased activity levels observed in dementia groups compared } \\
\text { to controls. Increased stressed and resting heart rates in dementia } \\
\text { groups compared to controls. }\end{array}$ & 17 \\
\hline Anderson et al. (14) ${ }^{\ddagger}$ & $\begin{array}{l}\text { Prospective } \\
\text { case control }\end{array}$ & $\begin{array}{l}\text { FTD (13) } \\
\text { Control (11) }\end{array}$ & $\begin{array}{l}\text { FTD } 63.9(8.8) \\
\text { Control } \\
66.8(5.7)\end{array}$ & $\begin{array}{l}\text { Increase in nocturnal activity and decrease in morning activity in } \\
\text { dementia group compared to controls. No significant overall } \\
\text { difference in non-parametric analysis of circadian rhythm between } \\
\text { dementia group and controls. }\end{array}$ & 18 \\
\hline Brown et al. $(16)^{\ddagger}$ & $\begin{array}{l}\text { Prospective } \\
\text { cross sectional }\end{array}$ & $\begin{array}{l}\text { DEM AC (22) } \\
\text { Control (27) }\end{array}$ & Not Stated & $\begin{array}{l}\text { Less robust sleep wake rhythms, increased total sleep time, and } \\
\text { increased time spent in bed in group with dementia but no } \\
\text { difference in sleep efficiency as compared to participants without } \\
\text { dementia. }\end{array}$ & 17 \\
\hline Carvalho-Bos et al. (17) & $\begin{array}{l}\text { Prospective } \\
\text { cohort }\end{array}$ & $\begin{array}{l}\operatorname{AD}(57) \\
\operatorname{VaD}(13) \\
\operatorname{DEM} A C(10)\end{array}$ & $85.5(5.9)$ & $\begin{array}{l}\text { A lower level of cognitive functioning as measured by the MMSE } \\
\text { and higher functional impairment were associated with a less } \\
\text { stable rest-activity rhythm. }\end{array}$ & 17 \\
\hline David et al. (19) & $\begin{array}{l}\text { Prospective } \\
\text { Case control }\end{array}$ & $\begin{array}{l}\text { AD (32) } \\
\text { Control (15) }\end{array}$ & $\begin{array}{l}\text { AD } 78.6(7.4) \\
\text { Control } \\
73.1(6.0)\end{array}$ & Lower activity levels in dementia group compared to controls. & 13 \\
\hline David et al. (20) & $\begin{array}{l}\text { Prospective } \\
\text { cohort }\end{array}$ & $\mathrm{AD}(107)$ & AD $77.2(6.7)$ & $\begin{array}{l}\text { Participants with dementia and apathy had lower daytime activity } \\
\text { levels than those without apathy. }\end{array}$ & 16 \\
\hline Eggermont and Scherder (21) & $\begin{array}{l}\text { Prospective } \\
\text { cohort }\end{array}$ & DEM AC (76) & DEM AC 84.9 & No association between cognition and motor activity. & 17 \\
\hline Fetveit and Bjorvatn (22) & $\begin{array}{l}\text { Prospective } \\
\text { cross sectional }\end{array}$ & DEM AC (23) & $\begin{array}{l}\text { DEM AC } 86.1 \\
(7.0)\end{array}$ & $\begin{array}{l}\text { Consistent association between decreased cognition as measured } \\
\text { by the MMSE and reduced activity level as well as fragmented } \\
\text { sleep. }\end{array}$ & 18 \\
\hline Fleiner et al. (24) & $\begin{array}{l}\text { Prospective } \\
\text { cross sectional }\end{array}$ & DEM AC (45) & DEM AC 79 (7) & $\begin{array}{l}\text { Low activity levels observed with a wide range of activity patterns } \\
\text { in groups with dementia. }\end{array}$ & 16 \\
\hline Gehrman et al. (26) & $\begin{array}{l}\text { Retrospective } \\
\text { Cross sectional }\end{array}$ & DEM AC (150) & $\begin{array}{l}\text { DEM AC } 84.1 \\
(7.8)\end{array}$ & $\begin{array}{l}\text { No association between rest activity rhythm and severity of } \\
\text { dementia as measured by the MMSE, but changes in circadian } \\
\text { rhythm observed in those with dementia. }\end{array}$ & 16 \\
\hline Ghali et al. (27) & $\begin{array}{l}\text { Prospective } \\
\text { cohort }\end{array}$ & $A D(18)$ & AD $78.8(6.4)$ & $\begin{array}{l}\text { Time of nocturnal activity peak levels associated with duration of } \\
\text { illness (measured in years) in groups with dementia. }\end{array}$ & 16 \\
\hline Harper et al. $(28)^{\ddagger}$ & $\begin{array}{l}\text { Prospective } \\
\text { case control }\end{array}$ & $\begin{array}{l}\text { AD (32) } \\
\text { Control (8) }\end{array}$ & $\begin{array}{l}\text { AD } 70.2(1.0) \\
\text { Control } \\
72.8(2.1)\end{array}$ & $\begin{array}{l}\text { Increasing AD pathology associated with greater disturbances in } \\
\text { circadian activity. Difference in rest-activity between dementia and } \\
\text { control groups. }\end{array}$ & 17 \\
\hline Harper et al. $(29)^{\ddagger}$ & $\begin{array}{l}\text { Prospective } \\
\text { case control }\end{array}$ & $\begin{array}{l}\text { DEM AC (38) } \\
\text { Control (8) }\end{array}$ & $\begin{array}{l}\text { DEM AC } 70.2 \\
(1.0) \\
\text { Control } \\
72.8(2.1)\end{array}$ & $\begin{array}{l}\text { Increased nocturnal activity with circadian phase delay observed } \\
\text { in participants with } A D \text { compared to controls. }\end{array}$ & 15 \\
\hline Hatfield et al. $(30)^{\ddagger}$ & $\begin{array}{l}\text { Prospective } \\
\text { cross sectional }\end{array}$ & $\begin{array}{l}\text { AD (27) } \\
\text { Control (19) }\end{array}$ & $\begin{array}{l}\text { AD } 68.5(60-82) \\
\text { Control } \\
71.8(1.2)\end{array}$ & $\begin{array}{l}\text { Moderately demented participants show rest activity cycle } \\
\text { disturbance when compared to controls. No correlation seen } \\
\text { between severity of dementia as measured by the MMSE and } \\
\text { rest-activity rhythm. }\end{array}$ & 14 \\
\hline Hooghiemstra et al. $(31)^{\ddagger}$ & $\begin{array}{l}\text { Prospective } \\
\text { cross sectional }\end{array}$ & $\begin{array}{l}\text { DEM AC (61) } \\
\text { Control (68) }\end{array}$ & $\begin{array}{l}\text { DEM AC Median } \\
62.5 \\
\text { Control } \\
\text { Median } 63.0\end{array}$ & $\begin{array}{l}\text { More rest-activity rhythm fragmentation, more time in bed, more } \\
\text { time to transition from wake to sleep in those with early onset } \\
\text { dementia than controls. }\end{array}$ & 15 \\
\hline ljmker and Lamoth (35) & $\begin{array}{l}\text { Prospective } \\
\text { case control }\end{array}$ & $\begin{array}{l}\text { DEM AC (15) } \\
\text { Control (26) }\end{array}$ & $\begin{array}{l}\text { DEM AC } 81.7 \\
(6.3) \\
\text { Control } 70.6\end{array}$ & Changes in gait acceleration in dementia compared to controls & 13 \\
\hline Iwata et al. (36) & $\begin{array}{l}\text { Prospective } \\
\text { case control }\end{array}$ & $\begin{array}{l}\text { DEM AC (14) } \\
\text { Control (16) }\end{array}$ & $\begin{array}{l}\text { DEM AC } 74.8 \\
\text { Control } 73.7\end{array}$ & $\begin{array}{l}\text { Decreased physical activity in female subjects with dementia as } \\
\text { compared to controls }\end{array}$ & 14 \\
\hline James et al. (37) & $\begin{array}{l}\text { Retrospective } \\
\text { cross-sectional }\end{array}$ & $\begin{array}{l}\text { DEM AC (70) } \\
\text { Control (624) }\end{array}$ & Not stated & Lower levels of total daily activity in subjects with dementia & 16 \\
\hline
\end{tabular}


TABLE 6 | Continued

\begin{tabular}{|c|c|c|c|c|c|}
\hline Source & Study design & Participants ( $n$ ) & $\begin{array}{l}\text { Age, mean (SD } \\
\text { or range), y }\end{array}$ & Major findings & $\begin{array}{l}\text { Quality } \\
\text { score }^{\dagger}\end{array}$ \\
\hline Kodama et al. (38) & $\begin{array}{l}\text { Prospective } \\
\text { case control }\end{array}$ & $\begin{array}{l}\text { DEM AC (52) } \\
\text { Control (66) }\end{array}$ & $\begin{array}{l}\text { DEM AC } 78.5 \\
(10.7) \\
\text { Control } \\
72.4(6.7)\end{array}$ & $\begin{array}{l}\text { Circadian rhythm parameters significantly differed in subjects with } \\
\text { dementia compared to controls }\end{array}$ & 17 \\
\hline König et al. (39) & $\begin{array}{l}\text { Prospective } \\
\text { case control }\end{array}$ & $\begin{array}{l}\text { AD (23) } \\
\text { Control (22) }\end{array}$ & $\begin{array}{l}\text { AD } 77(9) \\
\text { Control } 73(7)\end{array}$ & $\begin{array}{l}\text { A difference in gait speed under dual task conditions was } \\
\text { observed between dementia subjects and controls }\end{array}$ & 17 \\
\hline Kuhlmei et al. $(41)^{\ddagger}$ & $\begin{array}{l}\text { Retrospective } \\
\text { cross-sectional }\end{array}$ & $\begin{array}{l}\text { DEM AC (32) } \\
\text { Control (23) }\end{array}$ & $\begin{array}{l}\text { DEM AC } 81 \\
\text { Control } 78\end{array}$ & Reduced daytime activity levels seen in subjects with dementia & 12 \\
\hline La Morgia et al. $(43)^{\ddagger}$ & $\begin{array}{l}\text { Prospective } \\
\text { case control }\end{array}$ & $\begin{array}{l}\text { AD (16) } \\
\text { Control (10) }\end{array}$ & $\begin{array}{l}\text { AD } 70.2(10.2) \\
\text { Control } \\
65.8(7.5)\end{array}$ & $\begin{array}{l}\text { Reduced sleep efficiency seen in subjects with AD as compared to } \\
\text { controls }\end{array}$ & 14 \\
\hline Lamoth et al. (44) & $\begin{array}{l}\text { Prospective } \\
\text { case control }\end{array}$ & $\begin{array}{l}\text { AD (13) } \\
\text { Control (13) }\end{array}$ & $\begin{array}{l}\text { AD } 82.6(4.2) \\
\text { Control } \\
79.3(5.5)\end{array}$ & Changes in gait variability in AD compared to controls & 16 \\
\hline Landolt et al. (45) & $\begin{array}{l}\text { Prospective } \\
\text { case control }\end{array}$ & sCJD (7) & sCJD 65.8 (3.8) & High frequency of sleep wake changes seen in those with sCJD. & 15 \\
\hline Lee et al. $(46)^{\ddagger}$ & $\begin{array}{l}\text { Prospective } \\
\text { case control }\end{array}$ & $\begin{array}{l}\text { AD (7) } \\
\text { Control (11) }\end{array}$ & $\begin{array}{l}\text { AD } 77.0(4.3) \\
\text { Control } \\
74.2(5.2)\end{array}$ & $\begin{array}{l}\text { Mean phase difference (MESOR) was different between those with } \\
\text { AD and controls. No significant change was seen in mean } \\
\text { acrophase or mean amplitude of temperature. }\end{array}$ & 15 \\
\hline Leger et al. (47) & $\begin{array}{l}\text { Retrospective } \\
\text { cross sectional }\end{array}$ & AD (208) & AD $73(11.6)$ & $\begin{array}{l}\text { Increased time spent in bed in those with moderate AD as } \\
\text { measured by the MMSE compared to those with mild AD. }\end{array}$ & 16 \\
\hline McCurry et al. (49) & $\begin{array}{l}\text { Prospective } \\
\text { cohort }\end{array}$ & $\mathrm{AD}(44)$ & AD $78.8(7.2)$ & $\begin{array}{l}\text { Significant variation seen in all sleep measures both between and } \\
\text { within all subjects }\end{array}$ & 14 \\
\hline Merrilees et al. (50) & $\begin{array}{l}\text { Prospective } \\
\text { cohort }\end{array}$ & FTD (22) & FTD 63.8 & $\begin{array}{l}\text { In patients with FTD, apathy was associated with lower activity } \\
\text { levels and greater number of bouts of immobility }\end{array}$ & 15 \\
\hline Most et al. (52) $)^{\ddagger}$ & $\begin{array}{l}\text { Prospective } \\
\text { case control }\end{array}$ & $\begin{array}{l}\text { AD (55) } \\
\text { Control (26) }\end{array}$ & $\begin{array}{l}\text { AD } 70.4(3.2) \\
\text { Control } \\
73.0(4.4)\end{array}$ & $\begin{array}{l}\text { Longer sleep onset latency and decreased sleep efficiency was } \\
\text { seen in subjects with AD compared to controls. }\end{array}$ & 15 \\
\hline Moyle et al. (53) & $\begin{array}{l}\text { Retrospective } \\
\text { cross sectional }\end{array}$ & DEM AC (192) & $\begin{array}{l}\text { DEM AC } 85.5 \\
(7.7)\end{array}$ & $\begin{array}{l}\text { No significant correlation seen between level of cognitive } \\
\text { impairment as measured by the MMSE and activity and sleep } \\
\text { patterns over } 24 \mathrm{~h} \text {. }\end{array}$ & 16 \\
\hline Mulin et al. (54) & $\begin{array}{l}\text { Prospective } \\
\text { cohort }\end{array}$ & AD (103) & AD $76.9(7.2)$ & $\begin{array}{l}\text { Subjects with apathy demonstrated more time spent in bed during } \\
\text { the night, and lower daytime motor activity than those without } \\
\text { apathy. }\end{array}$ & 14 \\
\hline Murphy et al. (55) & $\begin{array}{l}\text { Retrospective } \\
\text { cross-sectional }\end{array}$ & DEM AC (20) & $\begin{array}{l}\text { DEM AC } 78.7 \\
(1.8)\end{array}$ & $\begin{array}{l}\text { Energy expenditure inversely related to time spent lying down and } \\
\text { sleep duration. }\end{array}$ & 15 \\
\hline Olsen et al. (56) & $\begin{array}{l}\text { Retrospective } \\
\text { cross-sectional }\end{array}$ & DEM AC (193) & DEM AC 83.6 & $\begin{array}{l}\text { Decreased activity in nursing home subjects with dementia } \\
\text { compared to home dwelling subjects with dementia. }\end{array}$ & 16 \\
\hline Paavilainen et al. $(57)^{\ddagger}$ & $\begin{array}{l}\text { Prospective } \\
\text { case control }\end{array}$ & $\begin{array}{l}\text { DEM AC (23) } \\
\text { Control (19) }\end{array}$ & $\begin{array}{l}\text { DEM AC } 84.3 \\
(9.5) \\
\text { Control } \\
81.5(9.0)\end{array}$ & $\begin{array}{l}\text { Subject with dementia demonstrated lower daytime and higher } \\
\text { nocturnal activity than controls }\end{array}$ & 16 \\
\hline Pollak and Stokes $(58)^{\ddagger}$ & $\begin{array}{l}\text { Prospective } \\
\text { case control }\end{array}$ & $\begin{array}{l}\text { DEM AC (25) } \\
\text { Control (18) }\end{array}$ & $\begin{array}{l}\text { DEM AC } 80.7 \\
(7.9) \\
\text { Control } \\
73.7(7.2)\end{array}$ & $\begin{array}{l}\text { Less activity and flat cosine analysis of circadian rhythm in groups } \\
\text { with dementia when compared to controls }\end{array}$ & 18 \\
\hline $\begin{array}{l}\text { Rindlisbacher and Hopkins } \\
\text { (60) }\end{array}$ & $\begin{array}{l}\text { Prospective } \\
\text { cohort }\end{array}$ & $\mathrm{AD}(12)$ & AD 79.4 & Variability in 24-h peaks of activity correlated with years of illness & 16 \\
\hline Schwenk et al. (61) & $\begin{array}{l}\text { Prospective } \\
\text { cohort }\end{array}$ & DEM AC (77) & $\begin{array}{l}\text { DEM AC } 81.8 \\
(6.3)\end{array}$ & $\begin{array}{l}\text { Actigraph derived "walking bouts average duration" demonstrated } \\
\text { a positive predictive value for future falls in subjects with dementia }\end{array}$ & 17 \\
\hline Valembois et al. (62) $)^{\ddagger}$ & $\begin{array}{l}\text { Prospective } \\
\text { cross sectional }\end{array}$ & $\begin{array}{l}\text { DEM AC (126) } \\
\text { Control (57) }\end{array}$ & $\begin{array}{l}\text { All Participants } \\
84.9(6.8)\end{array}$ & $\begin{array}{l}\text { Decreased motor activity in subjects with dementia who } \\
\text { demonstrate apathy and anxiety. No association between agitation } \\
\text { and motor activity. }\end{array}$ & 15 \\
\hline Van Alphen et al. (63) & $\begin{array}{l}\text { Retrospective } \\
\text { cross-sectional }\end{array}$ & DEM AC (146) & $\begin{array}{l}\text { DEM AC } 83.0 \\
(7.6)\end{array}$ & $\begin{array}{l}\text { Increased sedentary levels and decreased physical activity levels } \\
\text { in subjects with dementia who were institutional dwelling }\end{array}$ & 17 \\
\hline van Someren et al. $(64)^{\ddagger}$ & $\begin{array}{l}\text { Prospective } \\
\text { case control }\end{array}$ & $\begin{array}{l}\text { DEM AC (34) } \\
\text { Control (11) }\end{array}$ & $\begin{array}{l}\text { DEM AC } 74.7 \\
\text { Control } 72(1.2)\end{array}$ & $\begin{array}{l}\text { Less stable rest-activity rhythm in institutionalized subjects with } \\
\text { dementia compared to subjects cared for at home and controls }\end{array}$ & 15 \\
\hline
\end{tabular}


TABLE 6 | Continued

\begin{tabular}{|c|c|c|c|c|c|}
\hline Source & Study design & Participants (n) & $\begin{array}{l}\text { Age, mean (SD } \\
\text { or range), y }\end{array}$ & Major findings & $\begin{array}{l}\text { Quality } \\
\text { score }^{\dagger}\end{array}$ \\
\hline Varma and Watts $(65)^{\ddagger}$ & $\begin{array}{l}\text { Prospective } \\
\text { case control }\end{array}$ & $\begin{array}{l}\text { AD (39) } \\
\text { Control (53) }\end{array}$ & $\begin{array}{l}\text { AD } 73.5(7.9) \\
\text { Control } \\
73.2(6.5)\end{array}$ & $\begin{array}{l}\text { Decreased physical activity and changes in activity patterns seen } \\
\text { in dementia subjects compared to controls }\end{array}$ & 15 \\
\hline Viegas et al. (66) & $\begin{array}{l}\text { Retrospective } \\
\text { cross sectional }\end{array}$ & DEM AC (104) & $\begin{array}{l}\text { DEM AC } 82.9 \\
(8.4)\end{array}$ & Average of $476 \mathrm{~min}$ sleep per $24 \mathrm{~h}$ in subjects with dementia. & 18 \\
\hline Volicer et al. $(67)^{\ddagger}$ & $\begin{array}{l}\text { Prospective } \\
\text { case control }\end{array}$ & $\begin{array}{l}\text { AD (25) } \\
\text { Control (9) }\end{array}$ & $\begin{array}{l}\text { AD } 71.0(60-88) \\
\text { Control } \\
73.4(67-83)\end{array}$ & $\begin{array}{l}\text { A high percentage of nocturnal activity and less diurnal motor } \\
\text { activity in subjects with } A D \text { compared to controls }\end{array}$ & 17 \\
\hline Wams et al. $(68)^{\ddagger}$ & $\begin{array}{l}\text { Retrospective } \\
\text { cross sectional }\end{array}$ & $\begin{array}{l}\text { AD (29) } \\
\text { Control (14) }\end{array}$ & $\begin{array}{l}\text { AD } 77.7(7.6) \\
\text { Control } \\
73.8(4.6)\end{array}$ & $\begin{array}{l}\text { AD patients demonstrated longer time in bed, longer sleep } \\
\text { duration, and lower amplitude than controls. No difference } \\
\text { between groups in sleep quality }\end{array}$ & 17 \\
\hline Weissová et al. (69) & $\begin{array}{l}\text { Prospective } \\
\text { case control }\end{array}$ & $\begin{array}{l}\mathrm{AD}(4) \\
\text { Control (4) }\end{array}$ & Not stated & $\begin{array}{l}\text { No difference in sleep parameters in participants with AD } \\
\text { compared to controls }\end{array}$ & 16 \\
\hline Wirz-Justice et al. $(70)^{\ddagger}$ & $\begin{array}{l}\text { Prospective } \\
\text { cross sectional }\end{array}$ & $\begin{array}{l}\mathrm{KP}(6) \\
\text { Control (6) }\end{array}$ & $\begin{array}{l}\text { KP } 66.8 \\
\text { Control } \\
\text { Not Stated }\end{array}$ & $\begin{array}{l}\text { Longer nocturnal rest duration and lower daytime activity level in } \\
\text { participants with KP compared to controls }\end{array}$ & 14 \\
\hline Yesavage et al. (71) & $\begin{array}{l}\text { Retrospective } \\
\text { cross sectional }\end{array}$ & $\mathrm{AD}(61)$ & AD $71.4(8.1)$ & $\begin{array}{l}\text { AD participants show worsening in parameters of nocturnal sleep } \\
\text { but no change in rest/activity circadian rhythm over time }\end{array}$ & 13 \\
\hline
\end{tabular}

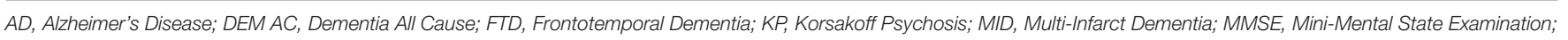
sCJD, sporadic Creutzfeldt-Jakob Disease; SD, Standard Deviation; VaD, Vascular Dementia; y, Years.

${ }^{\dagger}$ Refers to endpoints reported by authors.

¥Study included in one or multiple meta-analyses.

TABLE 7 | Combined effect estimates and heterogeneity for actigraphy outcomes between dementia and control samples.

\begin{tabular}{|c|c|c|c|c|c|c|}
\hline $\begin{array}{l}\text { Actigraphy } \\
\text { measure }\end{array}$ & $\begin{array}{l}\text { Included } \\
\text { studies, no. }\end{array}$ & $\begin{array}{c}\text { Dementia } \\
\text { subjects, no. }\end{array}$ & $\begin{array}{c}\text { Healthy } \\
\text { subjects, no. }\end{array}$ & $\begin{array}{c}\text { Pooled mean } \\
\text { difference, } \\
\text { random-effects model } \\
(95 \% \mathrm{Cl})\end{array}$ & $Q$ & $I^{2}(95 \% \mathrm{Cl})$ \\
\hline IS & 10 & 298 & 180 & $-0.47(-0.81,-0.14)^{\dagger}$ & $24.86(9 d f)^{\S}$ & $64(29,82)$ \\
\hline IV & 9 & 245 & 171 & $0.46(0.27,0.65)^{\dagger}$ & $6.61(8 d f)$ & $0(0,65)$ \\
\hline RA & 9 & 237 & 152 & $-0.53(-0.76,-0.30)^{\dagger}$ & $15.39(8 d f)$ & $48(0,76)$ \\
\hline M10 & 8 & 184 & 103 & $-0.61(-1.16,-0.06)^{\S}$ & $30.32(7 d f)^{\dagger}$ & $77(54,88)$ \\
\hline TST & 7 & 291 & 212 & $0.46(-0.21,1.12)$ & $30.31(6 d f)^{\dagger}$ & $80(60,90)$ \\
\hline SE & 8 & 193 & 171 & $-0.52(-0.89,-0.16)^{\S}$ & $15.44(7 d f)^{\S}$ & $55(0,80)$ \\
\hline Amplitude & 7 & 174 & 93 & $-1.22(-1.94,-0.50)^{\ddagger}$ & $36.34(6 d f)^{\dagger}$ & $83(67,92)$ \\
\hline Daytime Activity & 7 & 210 & 136 & $-1.60(-2.66,-0.55)^{\S}$ & $81.82(6 d f)^{\dagger}$ & $93(87,96)$ \\
\hline
\end{tabular}

df, degrees of freedom; IS, interdaily stability; IV, intradaily variability; $I^{2}$, percentage of variation across studies due to heterogeneity; $L 5$, activity of least active $5 \mathrm{~h}$; M10, activity of most active $10 \mathrm{~h}$; No., number; $Q$, Cochran's $Q$ weighted sum of squares of effect size estimates subtracted by their mean; RA, relative amplitude; SE, sleep efficiency; TST, total sleep time. $t_{p}<0.0001$.

$\neq_{p}<0.001$

$\S_{p}<0.05$.

\section{Risk of Publication Bias Across Studies for Meta-Analysis}

Funnel plots for each variable investigated using random effects meta-analysis are provided in Figure 3. These plots were constructed with a measure of study size on the $\mathrm{x}$-axis and a measure of effect size on the y-axis. Dashed lines represent the pseudo 95 and $99.7 \%$ confidence limits about the effect estimate (solid line). Funnel plot asymmetry was observed for all but two variables (IV and RA), and significant Egger tests observed for M10 $(p=0.0057)$ and amplitude variables $(p=0.0078)$, suggesting evidence of publication bias for these measurements.

\section{Investigation of Influential Studies}

The impact of each study on a meta-analysis estimate was investigated through influence analysis. Influence analysis shows that meta-analysis estimates are generally robust (Figure 4), excluding meta-analysis of daytime activity, where the pooled estimate decreases in magnitude markedly and precision of the estimate improves with exclusion of Varma and Watts (65). Even with exclusion of this influential study, the pooled estimate remains significant and shows the same direction of effect as in the full meta-analysis. 
A

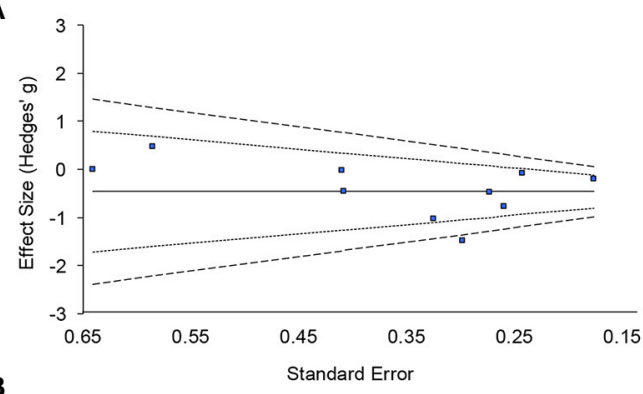

B

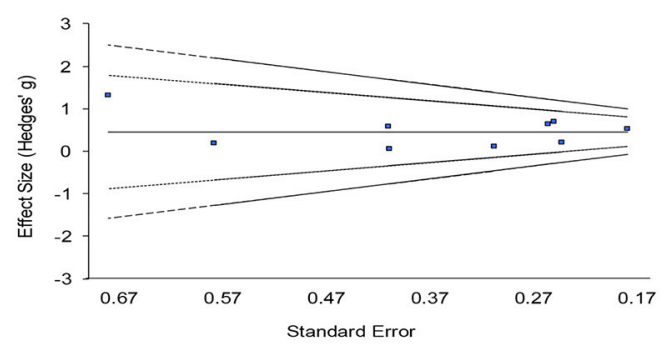

C

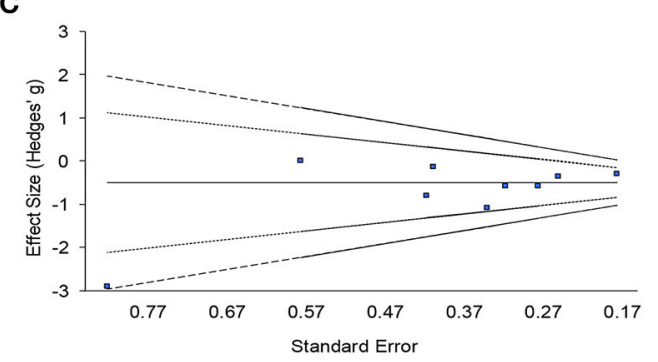

G

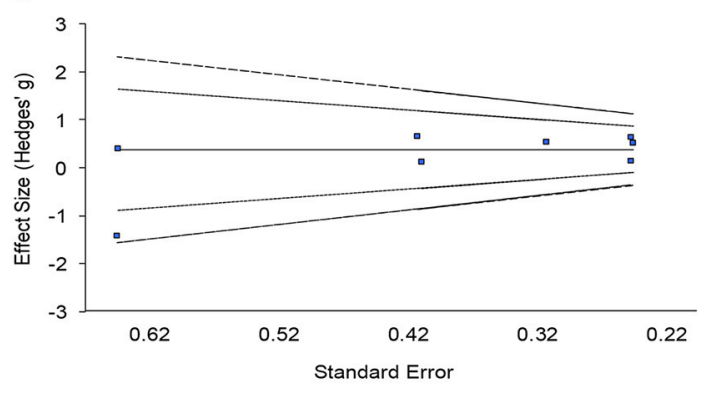

H

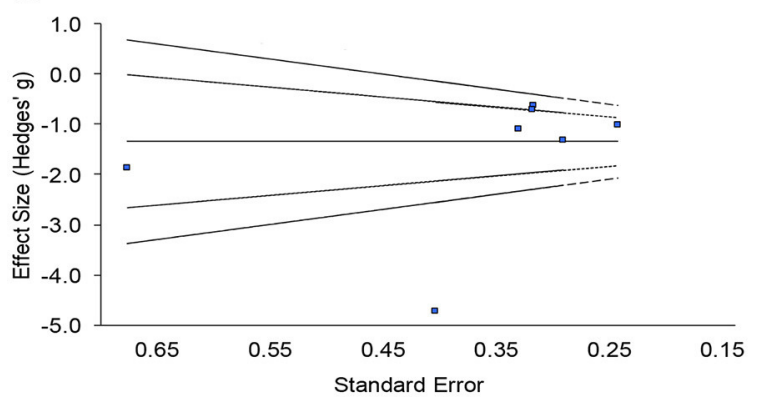

D

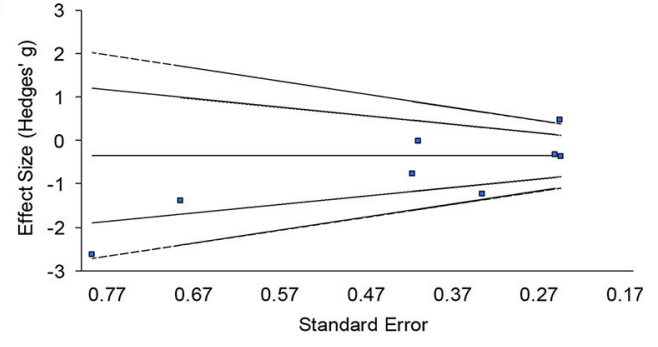

E
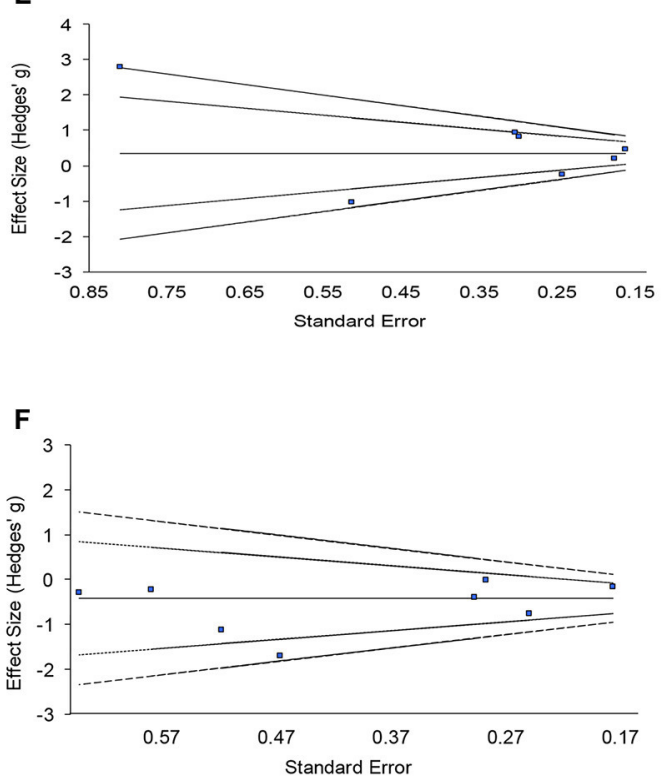

I

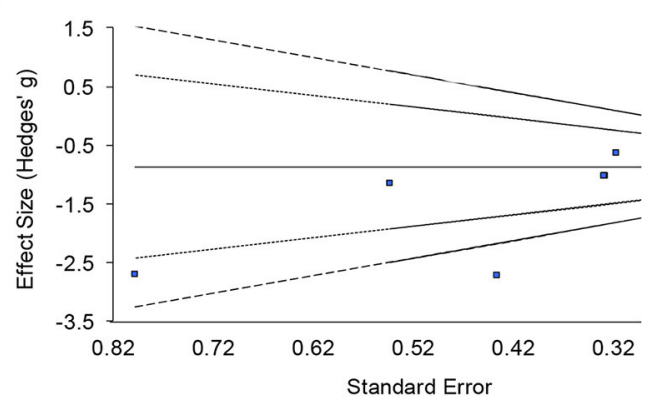

FIGURE 3 | Funnel plots with pseudo 95 and 99.7\% confidence intervals assessing publication bias of included studies for nine actigraphy measures. (A) Interdaily stability, (B) intradaily variability, (C) relative amplitude, (D) activity of most active 10h, (E) total sleep time, (F) sleep efficiency, (G) activity of least active 5 h, (H) daytime activity, (I) amplitude. 


A
\begin{tabular}{|c|c|}
\hline Study omitted & Estimate [95\% Cl] \\
\hline Anderson et al. 2009 & $-0.70[-1.19,-0.22]$ \\
\hline Harper et al. 2004 & $-0.56[-1.03,-0.10]$ \\
\hline La Morgia et al. 2016 & $-0.65[-1.14,-0.16]$ \\
\hline Most et al. 2012 & $-0.72[-1.23,-0.21]$ \\
\hline Van Someren et al. 1996 & $-0.57[-1.04,-0.11]$ \\
\hline Volicer et al. 2001 & $-0.47[-0.85,-0.09]$ \\
\hline Wirz-Justice et al. 2010 & $-0.72[-1.17,-0.27]$ \\
\hline Hatfield et al. 2004 & $-0.55[-1.00,-0.09]$ \\
\hline Hooghiemstra et al. 2015 & $-0.71[-1.24,-0.18]$ \\
\hline Wams et al. 2017 & $-0.63[-1.07,-0.19]$ \\
\hline
\end{tabular}

B

\begin{tabular}{|c|c|}
\hline Study omitted & Estimate [95\% Cl] \\
\hline Anderson et al. 2009 & $0.48[0.26,0.71]$ \\
\hline Harper et al. 2004 & $0.49[0.27,0.71]$ \\
\hline La Morgia et al. 2016 & $0.44[0.19,0.69]$ \\
\hline Most et al. 2012 & $0.52[0.28,0.76]$ \\
\hline Van Someren et al. 1996 & $0.43[0.19,0.68]$ \\
\hline Wirz-Justice et al. 2010 & $0.47[0.23,0.70]$ \\
\hline Hatfield et al. 2004 & $0.40[0.18,0.62]$ \\
\hline Hooghiemstra et al. 2015 & $0.42[0.13,0.71]$ \\
\hline Wams et al. 2017 & $0.43[0.22,0.65]$ \\
\hline
\end{tabular}

C

\begin{tabular}{|c|c|}
\hline Study omitted & Estimate $[95 \%$ Cl] \\
\hline Anderson et al. 2009 & $-0.65[-1.04,-0.26]$ \\
\hline Harper et al. 2004 & $-0.62[-1.02,-0.21]$ \\
\hline Harper et al. 2001 & $-0.62[-1.02,-0.23]$ \\
\hline La Morgia et al. 2016 & $-0.57[-0.96,-0.17]$ \\
\hline Volicer et al. 2001 & $-0.51[-0.87,-0.15]$ \\
\hline Wirz-Justice et al. 2010 & $-0.64[-1.02,-0.26]$ \\
\hline Hatfield et al. 2004 & $-0.57[-0.97,-0.17]$ \\
\hline Hooghiemstra et al. 2015 & $-0.67[-1.09,-0.25]$ \\
\hline Wams et al. 2017 & $-0.45[-0.68,-0.21]$ \\
\hline
\end{tabular}

FIGURE 4 | Continued

\section{DISCUSSION}

From our systematic review of the literature we found 48 articles which met our inclusion criteria of wearable technology use in patients with dementia for the measurement of physiological parameters. Wearable devices were utilized most extensively to
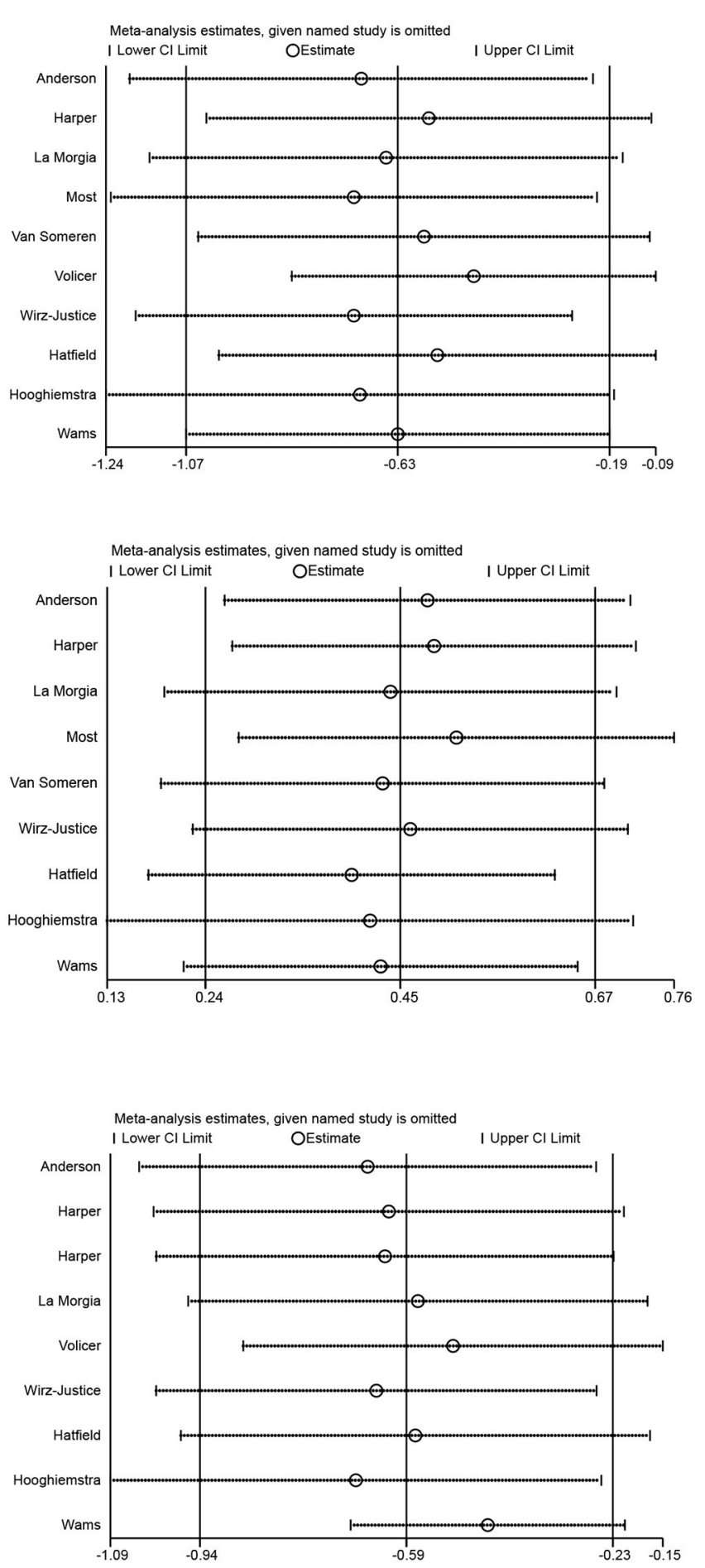
D

\begin{tabular}{|c|c|}
\hline Study omitted & Estimate [95\% Cl] \\
\hline Anderson et al. 2009 & $-0.84[-1.51,-0.17]$ \\
\hline Harper et al. 2004 & $-0.68[-1.33,-0.025]$ \\
\hline La Morgia et al. 2016 & $-0.72[-1.40,-0.05]$ \\
\hline Most et al. 2012 & $-0.86[-1.29,-0.43]$ \\
\hline Van Someren et al. 1996 & $-0.68[-1.34,-0.025]$ \\
\hline Wirz-Justice et al. 2010 & $-0.55[-1.10,-0.0008]$ \\
\hline Hatfield et al. 2004 & $-0.73[-1.41,-0.041]$ \\
\hline Wams et al. 2017 & $-0.65[-1.27,-0.027]$ \\
\hline
\end{tabular}

E

\begin{tabular}{|c|c|}
\hline Study omitted & Estimate [95\% Cl] \\
\hline Aharon-Peretz et al. 1991 & $-0.30[-0.19,-0.80]$ \\
\hline Lee et al. 2004 & $0.50[0.07,0.93]$ \\
\hline Hooghiemstra et al. 2015 & $0.43[-0.17,1.03]$ \\
\hline Brown et al. 2015 & $0.28[-0.22,0.78]$ \\
\hline Valembois et al. 2015 & $0.37[-0.23,0.97]$ \\
\hline Wams et al. 2017 & $0.24[-0.16,0.63]$ \\
\hline Most et al. 2012 & $0.49[0.0068,0.98]$ \\
\hline
\end{tabular}

$\mathbf{F}$

\begin{tabular}{|c|c|}
\hline Study omitted & Estimate [95\% Cl] \\
\hline Aharon-Peretz et al. 1991 & $-0.55[-0.97,-0.13]$ \\
\hline La Morgia et al. 2016 & $-0.31[-0.61,-0.01]$ \\
\hline Lee et al. 2004 & $-0.40[-0.80,0.0039]$ \\
\hline Most et al. 2012 & $-0.40[-0.84,0.034]$ \\
\hline Wirz-Justice et al. 2010 & $-0.49[-0.91,-0.06]$ \\
\hline Brown et al. 2015 & $-0.55[-1.00,-0.11]$ \\
\hline Hooghiemstra et al. 2015 & $-0.55[-1.02,-0.068]$ \\
\hline Wams et al. 2017 & $-0.48[-0.90,-0.06]$ \\
\hline
\end{tabular}
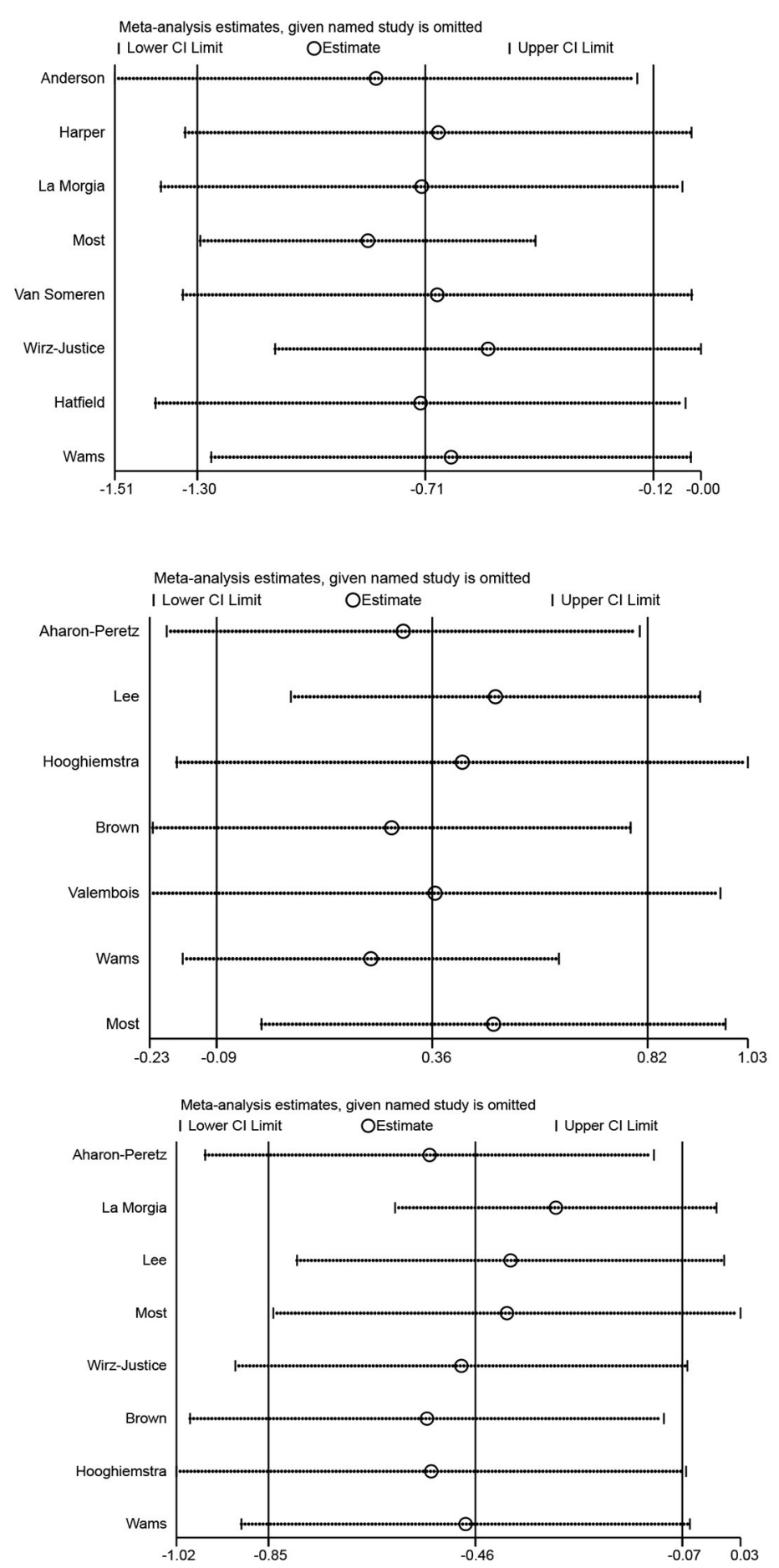

FIGURE 4 | Continued

systematic review and meta-analysis of wearable device testing in participants with dementia.

\section{Wearable Devices to Measure Sleep and Circadian Rhythm}

The use of actigraphy to measure sleep was the most commonly reported outcome. Participants with dementia demonstrated reduced sleep efficiency as compared to controls. There was also a significant difference between individuals with dementia and controls on non-parametric measures of circadian rhythm including IV, IS, and RA, however it should be noted that for some measures the combined effects were substantially weighted by the results of Hooghiemstra et al. (31). Meta-analysis of the amplitude measure of circadian 


\begin{tabular}{|c|c|}
\hline Study omitted & Estimate [95\% Cl] \\
\hline Anderson et al. 2009 & $0.39[-0.02,0.79]$ \\
\hline Harper et al. 2004 & $0.29[-0.10,0.68]$ \\
\hline La Morgia et al. 2016 & $0.31[-0.10,0.72]$ \\
\hline Most et al. 2012 & $0.28[-0.15,0.71]$ \\
\hline Van Someren et al. 1996 & $0.32[-0.10,0.74]$ \\
\hline Wirz-Justice et al. 2010 & $0.49[0.21,0.77]$ \\
\hline Hatfield et al. 2004 & $0.41[0.0022,0.81]$ \\
\hline Wams et al. 2017 & $0.35[-0.046,0.74]$ \\
\hline
\end{tabular}

H

\begin{tabular}{|c|c|}
\hline Study omitted & Estimate [95\% Cl] \\
\hline Harper et al. 2004 & $-1.74[-2.95,-0.53]$ \\
\hline Paavilainen et al. 2005 & $-1.78[-3.01,-0.56]$ \\
\hline Pollak et al. 1997 & $-1.77[-3.01,-0.53]$ \\
\hline Varma et al. 2017 & $-0.96[-1.26,-0.65]$ \\
\hline Volicer et al. 2001 & $-1.64[-2.86,-0.41]$ \\
\hline Wirz-Justice et al. 2010 & $-1.57[-2.72,-0.42]$ \\
\hline Kuhlmei et al. 2013 & $-1.69[-2.97,-0.42]$ \\
\hline
\end{tabular}

I

\begin{tabular}{|c|c|}
\hline Study omitted & Estimate [95\% CI] \\
\hline Harper et al. 2004 & $-1.13[-1.84,-0.42]$ \\
\hline Harper et al. 2001 & $-0.80[-1.33,-0.28]$ \\
\hline Lee et al. 2004 & $-1.06[-1.73,-0.38]$ \\
\hline Most et al. 2012 & $-1.24[-1.80,-0.68]$ \\
\hline Paavilainen et al. 2005 & $-1.09[-1.83,-0.36]$ \\
\hline Pollak et al. 1997 & $-1.18[-1.93,-0.43]$ \\
\hline Wirz-Justice et al. 2010 & $-0.90[-1.48,-0.32]$ \\
\hline
\end{tabular}
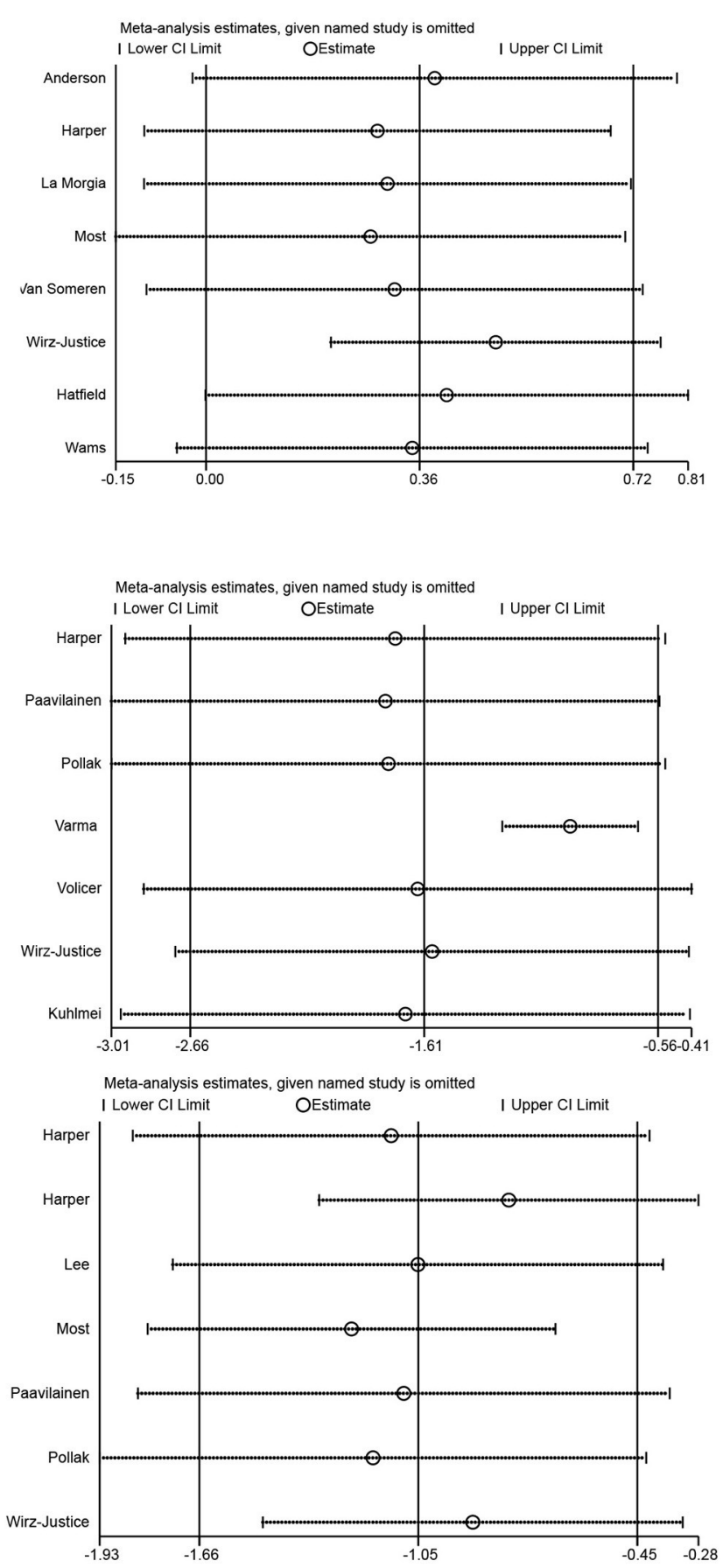

FIGURE 4 | Influence analysis. (A) Interdaily stability, (B) intradaily variability, (C) relative amplitude, (D) activity of most active $10 \mathrm{~h}$, (E) total sleep time, (F) sleep efficiency, (G) activity of least active 5 h, (H) daytime activity, and (I) amplitude.

rhythm cosinor analysis also demonstrated a moderate but statistically significant difference between groups. Again, a high level of heterogeneity between studies was observed for this outcome measure. Despite evidence of the utility of wearable actigraphy in sleep monitoring, consistent outcome measures and methods of analyzing sleep data and circadian rhythm have not been universally agreed upon (2). In order for actigraphy to become routinely used in clinical and drug treatment trials, consistent outcome measures are needed and, as shown in this meta-analysis, may provide a useful endpoint for patients with dementia.

\section{Wearable Devices and Daily Activity}

When using wearable devices to measure daily activity, those with dementia had significantly lower daily activity counts than controls. This effect was demonstrated despite acrossstudy variation in methods of calculating daytime activity including peak activity counts, mean activity, and daily activity. 
A meta-analysis of studies measuring daily activity showed that subjects with dementia demonstrate significantly less daily activity as compared to controls. Four groups reported no differences in nocturnal activity between subjects with dementia and controls. It should be noted that two of these studies did not recruit a control group, but instead compared participants with dementia to their caregivers [McCurry et al. (49) and Merrilees et al. (50)]. Physical activity has been examined in longitudinal studies and found to be associated with both development of dementia as well as disease progression (78). There is increasing evidence that physical activity and exercise as part of multidomain interventions holds benefit for patients with dementia (79). However, as demonstrated in this review, definitions of physical activity differ significantly between studies and daily activity counts measured by wearable devices are not definite indicators of beneficial exercise, but merely of movement. Some researchers have attempted to quantify daily activity counts into variables such as energy expenditure, and this measure was also reduced in participants with dementia as compared to controls (55). With the growing availability of consumer wrist worn devices for movement and activity tracking, the use of daily activity measurements provides a potential novel end point for large scale clinical trials in dementia.

\section{Wearable Devices and Gait}

Analysis of gait behavior was studied by six groups. Significant differences between controls and those with dementia were reported by all groups for multiple aspects of the gait cycle and behavior. However, due to the variation in reported outcomes, a quantitative analysis could not be performed and conclusions regarding the use of wearable devices for the study of gait could not be reliably made. It is important to note that gait speed and walking speed were reported as significantly different in subjects with dementia when compared to controls, while cadence and step variance were not. Lower gait speed in particular has been shown in numerous longitudinal studies to correlate with increased fall risk in older adults (80). Further work to replicate these findings in subjects with dementia is warranted.

\section{Limitations}

The main limitation of the meta-analysis was the between-study heterogeneity (Table 7). Given differences in characteristics of study design such as duration of testing, wearable device type, and diagnosis, statistical heterogeneity was expected between publications included in each meta-analysis. Despite this, effect size comparisons between healthy volunteers and participants with dementia were generally consistent in direction between studies. Methodological considerations specifically for actigraphy testing in dementia have been more thoroughly addressed in a clinical review (81). Also, all papers included in this review corresponded to definitions of both all cause dementia and wearable devices which were agreed upon by the author group. As

\section{REFERENCES}

1. Alzheimer's Disease International (ADI). Dementia: a Public Health Priority. World Health Organization (2013). a result, studies which did not conform to these definitions have been excluded and the effect these may have had on the analysis cannot be quantified. Lastly not all devices used have been compared to gold-standard clinical testing and their methods of measurement may differ and therefore their reported differences should be interpreted with caution.

\section{CONCLUSIONS AND IMPLICATIONS}

In conclusion this systematic review and meta-analysis has shown that the wearable devices studied demonstrate differences in those with dementia when compared to controls. Specifically, it provides evidence that wearable devices demonstrate a utility in measuring levels of activity, changes in circadian rhythm, and changes in the sleep wake cycle. Included studies were limited by their heterogeneity, the lack of classification of dementia sub-type and stage, as well as the lack of confirmatory clinical trials. Further work is warranted to correlate these findings with clinical changes which may represent surrogate digital end-points such as the neuro-psychiatric manifestations associated with circadian rhythm changes and the loss of mobility associated with decreased activity.

\section{DATA AVAILABILITY STATEMENT}

All datasets generated for this study are included in the article/Supplementary Material.

\section{AUTHOR CONTRIBUTIONS}

JB: concept and design. JB, AC, and RP: acquisition, analysis, or interpretation of data, and drafting of the manuscript. JB, AC, RP, NK, and KT: critical revision of the manuscript for important intellectual content, administrative, technical, or material support. AC: statistical analysis. KT: obtained funding. JB and KT: supervision. AC had full access to all of the data in the study and takes responsibility for the integrity of the data and the accuracy of the data analysis. All authors contributed to the article and approved the submitted version.

\section{ACKNOWLEDGMENTS}

We thank Alissa Link, Education and Information Services Librarian, for her assistance in the construction of database search terms.

\section{SUPPLEMENTARY MATERIAL}

The Supplementary Material for this article can be found online at: https://www.frontiersin.org/articles/10.3389/fmed. 2020.501104/full\#supplementary-material

2. Shelgikar AV, Anderson PF, Stephens MR. Sleep tracking, wearable technology, and opportunities for research and clinical care. Chest. (2016) 150:732-43. doi: 10.1016/j.chest.2016. 04.016 
3. Vasunilashorn S, Steinman BA, Liebig PS, Pynoos J. Aging in place: evolution of a research topic whose time has come. J Aging Res. (2012) 2012:1-6. doi: 10.1155/2012/120952

4. Espay AJ, Bonato P, Nahab F, Maetzler W, Horak F, Lang AE, et al. Technology in parkinson disease: challenges and opportunities. Mov Disord. (2017) 31:1272-82. doi: 10.1002/mds. 26642

5. de Bruin ED, Hartmann A, Uebelhart D, Murer K, Zijlstra W. Wearable systems for monitoring mobility-related activities in older people: a systematic review. Clin Rehabil. (2008) 22:878-95. doi: 10.1177/0269215508090675

6. McCleery J, Cohen DA, Sharpley AL. Pharmacotherapies for sleep disturbances in dementia. Cochrane Database Syst Rev. (2016) 11:CD009178. doi: 10.1002/14651858.CD009178.pub3

7. Aharon-Peretz J, Masiah A, Pillar T, Epstein R, Tzischinsky O, Lavie P. Sleepwake cycles in multi-infarct dementia and dementia of the Alzheimer type. Neurology. (1991) 41:1616-9. doi: 10.1212/WNL.41.10.1616

8. American Psychiatric Association. Diagnostic and Statistical Manual of Mental Disorders. 3rd ed, revised. Washington, DC (1987).

9. McKhann G, Drachman D, Folstein M, Katzman R, Price D, Stadlan EM. Report of the NINCDS-ADRDA work group under the auspices of department of health and human services task force on Alzheimer's disease. Neurology. (1984) 34:939-44. doi: 10.1212/WNL.34.7.939

10. Ahmed RM, Landin-Romero $\mathrm{R}$, Collet $\mathrm{TH}$, van der Klaauw AA, Devenney E, Henning E, et al. Energy expenditure in frontotemporal dementia: a behavioural and imaging study. Brain. (2017) 140:171-83. doi: 10.1093/brain/aww263

11. McKhann GM, Albert MS, Grossman M, Miller B, Dickson D, Trojanowski JQ, et al. Clinical and pathological diagnosis of frontotemporal dementia: report of the Work Group on Frontotemporal Dementia and Pick's Disease. Arch Neurol. (2001) 58:1803-9. doi: 10.1001/archneur.58.11.1803

12. Gorno-Tempini ML, Hillis AE, Weintraub S, Kertesz A, Mendez M, Cappa SF, et al. Classification of primary progressive aphasia and its variants. Neurology. (2011) 76:1006-14. doi: 10.1212/WNL.0b013e3182 $1103 \mathrm{e} 6$

13. Rascovsky K, Hodges JR, Knopman D, Mendez MF, Kramer JH, Neuhaus J, et al. Sensitivity of revised diagnostic criteria for the behavioural variant of frontotemporal dementia. Brain. (2011) 134:2456-77. doi: 10.1093/brain/awr179

14. Anderson KN, Hatfield C, Kipps C, Hastings M, Hodges JR. Disrupted sleep and circadian patterns in frontotemporal dementia. Eur J Neurol. (2009) 16:317-23. doi: 10.1111/j.1468-1331.2008.02414.x

15. Neary D. Overview of frontotemporal dementias and the consensus applied. Dement Geriatr Cogn Disord. (1999) 10:6-9. doi: 10.1159/000051205

16. Brown DT, Westbury JL, Schüz B. Sleep and agitation in nursing home residents with and without dementia. Int Psychogeriatrics. (2015) 27:1945-55. doi: $10.1017 /$ S1041610215001568

17. Carvalho-Bos SS, Riemersma-van der Lek RF, Waterhouse J, Reilly T, Van Someren EJ. Strong association of the rest - activity rhythm with well-being in demented elderly women. Am J Geriatr Psychiatry. (2007) 15:92-100. doi: 10.1097/01.JGP.0000236584.03432.dc

18. American Psychiatric Association. Diagnostic and Statistical Manual of Mental Disorders. 4th ed. Washington, DC (1994).

19. David R, Rivet A, Robert PH, Mailland V, Friedman L, Zeitzer JM, et al. Ambulatory actigraphy correlates with apathy in mild Alzheimer's disease. Dementia. (2010) 9:509-16. doi: 10.1177/1471301210381678

20. David R, Mulin E, Friedman L, Le Duff F, Cygankiewicz E, Deschaux O, et al. Decreased daytime motor activity associated with apathy in Alzheimer disease: an actigraphic study. Am J Geriatr Psychiatry. (2012) 20:806-14. doi: 10.1097/JGP.0b013e31823038af

21. Eggermont LHP, Scherder EJA. Ambulatory but sedentary : impact on cognition and the rest - activity rhythm in nursing home residents with dementia. J Gerontol B Psychol Sci Soc Sci. (2008) 63:279-87. doi: 10.1093/geronb/63.5.P279

22. Fetveit A, Bjorvatn B. Sleep duration during the 24-hour day is associated with the severity of dementia in nursing home patients. Int J Geriatr Psychiatry. (2006) 21:945-50. doi: 10.1002/gps.1587

23. Morris JC. The Clinical Dementia Rating (CDR): current version and scoring rules. Neurology. (1993) 43:2412-4. doi: 10.1212/WNL.43.11. 2412-a
24. Fleiner T, Haussermann P, Mellone S, Zijlstra W. Sensor-based assessment of mobility-related behavior in dementia: feasibility and relevance in a hospital context. Int Psychogeriatr. (2016) 28:1687-94. doi: $10.1017 /$ S1041610216001034

25. World Health Organization. The ICD-10 Classification of Mental and Behavioral Disorders: Clinical Descriptions and Diagnostic Guidelines. Geneva: World Health Organization (1997).

26. Gehrman P, Marler M, Martin JL, Shochat T, Corey-Bloom J, AncoliIsrael S. The relationship between dementia severity and rest/activity circadian rhythms. Neuropsychiatr Dis Treat. (2005) 1:155-63. doi: $10.2147 /$ nedt.1.2.155.61043

27. Ghali LM, Hopkins RW, Rindlisbacher P. Ghali. 1995 Temporal shifts.pdf. Int J Geriatr Psychiatry. (1995) 10:517-21. doi: 10.1002/gps.930100612

28. Harper DG, Stopa EG, McKee AC, Satlin A, Fish D, Volicer L. Dementia severity and lewy bodies affect circadian rhythms in Alzheimer disease. Neurobiol Aging. (2004) 25:771-81. doi: 10.1016/j.neurobiolaging.2003.04.009

29. Harper DG, Stopa EG, Mckee AC, Satlin A, Patricia C, Goldstein R, et al. Differential circadian rhythm disturbances in men with alzheimer disease and frontotemporal degeneration. Arch Gen Psychiatry. (2001) 58:353-60. doi: 10.1001/archpsyc.58.4.353

30. Hatfield CF, Herbert J, Van Someren EJW, Hodges JR, Hastings MH. Disrupted daily activity/rest cycles in relation to daily cortisol rhythms of home-dwelling patients with early Alzheimer's dementia. Brain. (2004) 127:1061-74. doi: 10.1093/brain/awh129

31. Hooghiemstra AM, Eggermont LHP, Scheltens P, Van Der Flier WM, Scherder EJA. The rest-activity rhythm and physical activity in early-onset dementia. Alzheimer Dis Assoc Disord. (2015) 29:45-9. doi: 10.1097/WAD.0000000000000037

32. American Psychiatric Association. Diagnostic and Statistical Manual of Mental Disorders. 5th ed. Washington, DC (2013).

33. McKeith IG, Dickson DW, Lowe J, Emre M, O’Brien JT, et al. Diagnosis and management of dementia with Lewy bodies third report of the DLB consortium. Neurology. (2005) 65:1863-72. doi: 10.1212/01.wnl.0000187889.17253.b1

34. Pohjasvaara T, Mäntylä R, Ylikoski R, Kaste M, Erkinjuntti T. Comparison of different clinical criteria (DSM-III, ADDTC, ICD-10, NINDSAIREN, DSM-IV) for the diagnosis of vascular dementia. Stroke. (2000) 31:2952-7.

35. IJmker T, Lamoth CJC. Gait and cognition: the relationship between gait stability and variability with executive function in persons with and without dementia. Gait Posture. (2012) 35:126-30. doi: 10.1016/j.gaitpost.2011.08.022

36. Iwata A, Kowa H, Tsuji S. Monitoring daily life activity shows less activity among female dementia patients. Neurol Clin Neurosci. (2013) 1:91-5. doi: 10.1111/j.2049-4173.2013.00029.x

37. James BD, Boyle PA, Bennett DA, Buchman AS. Total daily activity measured with actigraphy and motor function in community-dwelling older persons with and without dementia. Alzheimer Dis Assoc Disord. (2012) 26:238-45. doi: 10.1097/WAD.0b013e31822fc3cb

38. Kodama A, Kume Y, Tsugaruya M, Ishikawa T. Deriving the reference value from the circadian motor active patterns in the "non-dementia" population, compared to the "dementia" population: what is the amount of physical activity conducive to the good circadian rhythm. Chronobiol Int. (2016) 33:1056-63. doi: 10.1080/07420528.2016.1196696

39. König A, Klaming L, Pijl M, Demeurraux A, David R, Robert P. Objective measurement of gait parameters in healthy and cognitively impaired elderly using the dual-task paradigm. Aging Clin Exp Res. (2017) 29:1181-9. doi: 10.1007/s40520-016-0703-6

40. Dubois B, Feldman HH, Jacova C, Hampel H, Molinuevo JL, Blennow $\mathrm{K}$, et al. Advancing research diagnostic criteria for Alzheimer's disease: the IWG-2 criteria. Lancet Neurol. (2014) 13:614-29. doi: 10.1016/S1474-4422(14)70090-0

41. Kuhlmei A, Walther B, Becker T, Müller U, Nikolaus T. Actigraphic daytime activity is reduced in patients with cognitive impairment and apathy. Eur Psychiatry. (2013) 28:94-7. doi: 10.1016/j.eurpsy.2011.04.006

42. Román GC, Tatemichi TK, Erkinjuntti T, Cummings JL, Masdeu JC, Garcia $\mathrm{JH}$, et al. Vascular Dementia Diagnostic criteria for research studies: Report of the NINDS-AIREN International Workshop. Neurology. (1993) 43:250-60. doi: 10.1212/WNL.43.2.250 
43. La Morgia C, Ross-Cisneros FN, Koronyo Y, Hannibal J, Gallassi R, Cantalupo $\mathrm{G}$, et al. Melanopsin retinal ganglion cell loss in Alzheimer disease. Ann Neurol. (2016) 79:90-109. doi: 10.1002/ana.24548

44. Lamoth CJ, Van Deudekom FJ, Van Campen JP, Appels BA, De Vries OJ, Pijnappels M. Gait stability and variability measures show effects of impaired cognition and dual tasking in frail people. J Neuroeng Rehabil. (2011) 8:2. doi: 10.1186/1743-0003-8-2

45. Landolt H, Glatzel M, Bla T, Achermann P, Roth C, Mathis J, et al. Sleepwake disturbances in sporadic Creutzfeldt-Jakob disease. Neurology. (2006) 66:1418-24. doi: 10.1212/01.wnl.0000210445.16135.56

46. Lee JH, Friedland R, Whitehouse PJ, Woo JI. Rhythms of sleep-wake cycle and temperature in alzheimer' s disease. J Neuropsychiatr. (2004) 16:192-8. doi: 10.1176/jnp.16.2.192

47. Leger D, Elbaz M, Dubois A, Rio S, Mezghiche H, Carita P, et al. Alzheimer's disease severity is not significantly associated with short sleep: survey by actigraphy on 208 mild and moderate alzheimer's disease patients. $J$ Alzheimers Dis. (2016) 55:321-31. doi: 10.3233/JAD-160754

48. Folstein MF, Folstein SE, McHugh PR. "Mini-mental state": a practical method for grading the cognitive state of patients for the clinician. J Psychiatr Res. (1975) 12:189-98.

49. McCurry SM, Pike KC, Vitiello MV, Logsdon RG, Teri L. Factors associated with concordance and variability of sleep quality in persons with Alzheimer's disease and their caregivers. Sleep. (2008) 31:741-8. doi: $10.1093 /$ sleep/31.5.741

50. Merrilees J, Dowling GA, Hubbard E, Mastick J, Ketelle R, Miller BL. Characterization of apathy in persons wth frontotemporal dementia and the impact on family caregivers. Alzheimer Dis Assoc Disord. (2012) 00:1-6. doi: 10.1097/WAD.0b013e3182471c54

51. Neary D, Snowden JS, Gustafson L, Passant U, Stuss D, Black S, et al. Frontotemporal lobar degeneration-a consensus on clinical diagnostic criteria. Neurology. (1998) 51:1546-54. doi: 10.1212/WNL.5 1.6 .1546

52. Most EIS, Aboudan S, Scheltens P, Van Someren EJW. Discrepancy between subjective and objective sleep disturbances in early- and moderate-stage alzheimer disease. Am J Geriatr Psychiatry. (2012) 20:460-7. doi: 10.1097/JGP.0b013e318252e3ff

53. Moyle W, Jones C, Murfield J, Draper B, Beattie E, Shum D, et al. Levels of physical activity and sleep patterns among older people with dementia living in long-term care facilities: A 24-h snapshot. Maturitas. (2017) 102:62-8. doi: 10.1016/j.maturitas.2017.05.015

54. Mulin E, Zeitzer JM, Friedman L, Le Duff F, Yesavage J, Robert PH, et al. Relationship between apathy and sleep disturbance in mild and moderate Alzheimer's disease: An actigraphic study. J Alzheimers Dis. (2011) 25:85-91. doi: 10.3233/JAD-2011-101701

55. Murphy J, Holmes J, Brooks C. Measurements of daily energy intake and total energy expenditure in people with dementia in care homes: the use of wearable technology. J Nutr Health Aging. (2017) 21:927-32. doi: 10.1007/s12603-017-0870-y

56. Olsen C, Pedersen I, Bergland A, Enders-Slegers M-J, Jøranson N, Calogiuri $\mathrm{G}$, et al. Differences in quality of life in home-dwelling persons and nursing home residents with dementia - a cross-sectional study. BMC Geriatr. (2016) 16:137. doi: 10.1186/s12877-016-0312-4

57. Paavilainen P, Korhonen I, Lötjönen J, Cluitmans L, Jylhä M, Särelä A, et al. Circadian activity rhythm in demented and non-demented nursing-home residents measured by telemetric actigraphy. J Sleep Res. (2005) 14:61-8. doi: 10.1111/j.1365-2869.2004.00433.x

58. Pollak CP, Stokes PE. Circadian rest-activity rhythms in demented and nondemented older community residents and their caregivers. J Am Geriatr Soc. (1997) 45:446-52. doi: 10.1111/j.1532-5415.1997.tb05169.x

59. Mattis. Mental status examination for organic mental syndrome in the elderly patient. In: Karasu E, editor. Geriatric Psychiatry. New York, NY: Grune and Stratton (1976)

60. Rindlisbacher P, \& Hopkins RW. An investigation of the sundowning syndrome. Int $J$ Geriatr Psychiatry. (1992) 7:15-23. doi: 10.1002/gps.930070104

61. Schwenk M, Hauer K, Zieschang T, Englert S, Mohler J, Najafi B. Sensorderived physical activity parameters can predict future falls in people with dementia. Gerontology. (2014) 60:483-92. doi: 10.1159/000363136
62. Valembois L, Oasi C, Pariel S, Jarzebowski W, Lafuente-Lafuente C, Belmin J. Wrist actigraphy: a simple way to record motor activity in elderly patients with dementia and apathy or aberrant motor behavior. J Nutr Heal Aging. (2015) 19:759-64. doi: 10.1007/s12603-015-0530-z

63. Van Alphen HJM, Volkers KM, Blankevoort CG, Scherder EJA, Hortobágyi T, Van Heuvelen MJG. Older adults with dementia are sedentary for most of the day. PLoS ONE. (2016) 11:1-16. doi: 10.1371/journal.pone.0152457

64. van Someren EJ, Hagebeuk EE, Lijzenga C, Scheltens P, de Rooij SE, Jonker C, et al. Circadian rest-activity rhythm disturbances in Alzheimer's disease. Biol Psychiatry. (1996) 40:259-70. doi: 10.1016/0006-3223(95)00370-3

65. Varma VR, Watts A. Daily physical activity patterns during the early stage of Alzheimer's disease. J Alzheimers Dis. (2017) 55:659-67. doi: 10.3233/JAD-160582

66. Viegas SM, Richards KC, Beck CK, Lambert CW, O’Sullivan PS, Cole CS, et al. Predictors of daytime sleep of nursing home residents with dementia. J Am Psychiatr Nurses Assoc. (2006) 12:286-93. doi: 10.1177/10783903062 95071

67. Volicer L, Harper DG, Manning BC, Goldstein R, Satlin A. Sundowning and circadian rhythms in alzheimer's disease. Am J Psychiatry. (2001) 158:704-11. doi: 10.1176/appi.ajp.158.5.704

68. Wams EJ, Wilcock GK, Foster RG, Wulff K. Sleep-wake patterns and cognition of older adults with amnestic mild cognitive impairment (aMCI): a comparison with cognitively healthy adults and moderate alzheimer's disease patients. Curr Alzheimer Res. (2017) 14:1030-41. doi: 10.2174/1567205014666170523095634

69. Weissová K, Bartoš A, Sládek M, Nováková M, Sumová A. Moderate changes in the circadian system of Alzheimer's disease patients detected in their home environment. PLOS ONE. (2016) 11:1-19. doi: 10.1371/journal.pone. 0146200

70. Wirz-Justice A, Schröder CM, Gasio PF, Cajochen C, Savaskan E. The circadian rest-activity cycle in korsakoff psychosis. Am J Geriatr Psychiatry. (2010) 18:33-41. doi: 10.1097/JGP.0b013e3181b0467a

71. Yesavage JA, Friedman L, Kraemer HC, Noda A, Wicks D, Bliwise DL, et al. A follow-up study of actigraphic measures in home-residing Alzheimer's disease patients. J Geriatr Psychiatry Neurol. (1998) 11:7-10. doi: 10.1177/089198879801100103

72. Lukowicz P, Kirstein T, Tröster G. Wearable systems for health care applications. Methods Inf Med. (2004) 43:232-8. doi: 10.1055/s-0038-1633863

73. Downs SH, Black N. The feasibility of creating a checklist for the assessment of the methodological quality both of randomised and non-randomised studies of health care interventions. J Epidemiol Commun Health. (1998) 52:377-84. doi: 10.1136/jech.52.6.377

74. Cohen J. Statistical Power Analysis for the Behavioral Sciences. Routledge (1988). Available from: https://www.taylorfrancis.com/books/9780203771587 (accessed September 8, 2018).

75. Borenstein M, Hedges LV, Higgins JPT, Rothstein HR. A basic introduction to fixed-effect and random-effects models for meta-analysis. Res Synth Methods. (2010) 1:97-111. doi: 10.1002/jrsm.12

76. Higgins JP, Green S. Cochrane Handbook for Systematic Reviews of Interventions: Cochrane Book Series. Chichester: The Cochrane Collaboration and John Wiley \& Sons Ltd. (2008). p. 1-649. doi: 10.1002/9780470712184

77. Ioannidis JPA. Perfect study, poor evidence: interpretation of biases preceding study design. Semin Hematol. (2008) 45:160-6. doi: 10.1053/j.seminhematol.2008.04.010

78. Rovio S, Kåreholt I, Viitanen M, Winblad B, Kivipelto M, Soininen $\mathrm{NH}$, et al. Leisure-time physical activity at midlife and the risk of dementia and Alzheimer's disease. Lancet Neurol. (2005). 4:705-11. doi: 10.1016/S1474-4422(05)70198-8

79. Ngandu T, Lehtisalo J, Solomon A, Levälahti E, Ahtiluoto S, Antikainen R, et al. A 2 year multidomain intervention of diet, exercise, cognitive training, and vascular risk monitoring versus control to prevent cognitive decline in at-risk elderly people (FINGER): a randomised controlled trial. Lancet. (2015) 385:2255-63. doi: 10.1016/S0140-6736(15)60461-5

80. Peel NM, Kuys SS, Klein K. Gait speed as a measure in geriatric assessment in clinical settings: a systematic review. J Gerontol A Biol Sci Med Sci. (2013) 68:39-46. doi: 10.1093/gerona/gls174

81. Camargos EF, Louzada FM, Nóbrega OT. Wrist actigraphy for measuring sleep in intervention studies with Alzheimer's 
$\begin{array}{lrrrr}\text { disease } & \text { patients: } & \text { application, usefulness, and challenges. } \\ \text { Sleep Med Rev. (2013) 17:475-88. doi: } & 10.1016 / \text { j.smrv.2013. }\end{array}$

01.006

Conflict of Interest: The authors declare that the research was conducted in the absence of any commercial or financial relationships that could be construed as a potential conflict of interest.
Copyright (C) 2021 Cote, Phelps, Kabiri, Bhangu and Thomas. This is an open-access article distributed under the terms of the Creative Commons Attribution License (CC $B Y)$. The use, distribution or reproduction in other forums is permitted, provided the original author(s) and the copyright owner(s) are credited and that the original publication in this journal is cited, in accordance with accepted academic practice. No use, distribution or reproduction is permitted which does not comply with these terms. 